Review

\title{
Natural Products from Physalis alkekengi L. var. franchetii (Mast.) Makino: A Review on Their Structural Analysis, Quality Control, Pharmacology, and Pharmacokinetics
}

\author{
Jing Yang ${ }^{1}$, Yanping Sun ${ }^{1}$, Feng Cao ${ }^{2}$, Bingyou Yang ${ }^{1}$ and Haixue Kuang ${ }^{1, *}$ \\ 1 Key Laboratory of Basic and Application Research of Beiyao, Ministry of Education, Heilongjiang University \\ of Chinese Medicine, Harbin 150040, China; mayday111@163.com (J.Y.); 18704608056@163.com (Y.S.); \\ ybywater@163.com (B.Y.) \\ 2 Ganjiang Chinese Medicine Innovation Center, Nanchang 330000, China; 18435166854@163.com \\ * Correspondence: hxkuang@hljucm.net; Tel.: +86-0451-82197188
}

check for updates

Citation: Yang, J.; Sun, Y.; Cao, F.; Yang, B.; Kuang, H. Natural Products from Physalis alkekengi L. var. franchetii (Mast.) Makino: A Review on Their Structural Analysis, Quality Control, Pharmacology, and

Pharmacokinetics. Molecules 2022, 27, 695. https://doi.org/10.3390/ molecules27030695

Academic Editor: Lars Porskjær Christensen

Received: 21 December 2021

Accepted: 17 January 2022

Published: 21 January 2022

Publisher's Note: MDPI stays neutral with regard to jurisdictional claims in published maps and institutional affiliations.

Copyright: (C) 2022 by the authors Licensee MDPI, Basel, Switzerland. This article is an open access article distributed under the terms and conditions of the Creative Commons Attribution (CC BY) license (https:// creativecommons.org/licenses/by/ $4.0 /)$

\begin{abstract}
The calyxes and fruits of Physalis alkekengi L. var. franchetii (Mast.) Makino (P. alkekengi), a medicinal and edible plant, are frequently used as heat-clearing and detoxifying agents in thousands of Chinese medicine prescriptions. For thousands of years in China, they have been widely used in clinical practice to treat throat disease, hepatitis, and bacillary dysentery. This systematic review summarizes their structural analysis, quality control, pharmacology, and pharmacokinetics. Furthermore, the possible development trends and perspectives for future research studies on this medicinal plant are discussed. Relevant information on the calyxes and fruits of P. alkekengi was collected from electronic databases, Chinese herbal classics, and Chinese Pharmacopoeia. Moreover, information was collected from ancient documents in China. The components isolated and identified in P. alkekengi include steroids, flavonoids, phenylpropanoids, alkaloids, nucleosides, terpenoids, megastigmane, aliphatic derivatives, organic acids, coumarins, and sucrose esters. Steroids, particularly physalins and flavonoids, are the major characteristic and bioactive ingredients in P. alkekengi. According to the literature, physalins are synthesized by the mevalonate and 2-C-methyl-D-erythritol-4-phosphate pathways, and flavonoids are synthesized by the phenylpropanoid pathway. Since the chemical components and pharmacological effects of P. alkekengi are complex and varied, there are different standards for the evaluation of its quality and efficacy. In most cases, the analysis was performed using high-performance liquid chromatography coupled with ultraviolet detection. A pharmacological study showed that the crude extracts and isolated compounds from P. alkekengi had extensive in vitro and in vivo biological activities (e.g., anti-inflammatory, anti-tumor, immunosuppressive, antibacterial, anti-leishmanial, anti-asthmatic, anti-diabetic, anti-oxidative, anti-malarial, anti-Alzheimer's disease, and vasodilatory). Moreover, the relevant anti-inflammatory and anti-tumor mechanisms were elucidated. The reported activities indicate the great pharmacological potential of P. alkekengi. Similarly, studies on the pharmacokinetics of specific compounds will also contribute to the progress of clinical research in this setting.
\end{abstract}

Keywords: the calyxes and fruits of P. alkekengi; structural analysis; quality control; pharmacology; pharmacokinetics

\section{Introduction}

P. alkekengi is a perennial plant (Figure 1a) belonging to the genus Physalis of the family Solanaceae. The calyxes and fruits of P. alkekengi (known as Jindenglong in Chinese) (Figure 1b) are distributed in Europe and Asia. The use of the calyxes and fruits of this plant was first recorded in the prestigious monograph Shennong Bencao Jing in China [1]. Subsequently, it was included as an important traditional Chinese medicine (TCM) in the Ben Cao Gang Mu and pharmacopoeia [2]. Calyxes are green, self-expanded into an oocyst shape, slightly concave at the base, $2.5-5 \mathrm{~cm}$ in length, $2.5-3.5 \mathrm{~cm}$ in diameter, have thin 
leathery skin, and are orange-red or fire-red when mature (Figure 1c). Fruits are spherical, orange-red, and 10-15 $\mathrm{mm}$ in diameter (Figure 1d). This plant has been used for $>2000$ years in China, and its activities have been defined as "heat-clearing and detoxifying, relieving sore throat to reducing phlegm and inducing diuresis for treating strangurtia" in TCM theory [3,4]. In clinical practice, P. alkekengi is often used in combination with other TCMs for the treatment of cough, excessive phlegm, pharyngitis, sore throat, dysuria, pemphigus, eczema, and jaundice [5]. Currently, the 12 TCM formulae and modern pharmaceutical preparations of the calyxes and fruits of P. alkekengi are listed in the Pharmacopoeia of the People's Republic of China and used in folk medicine [6]. For example, qing guo ointment, a TCM formula composed of seven medicinal herbal plants (i.e., the calyxes and fruits of $P$. alkekengi, Cannarii Fructus, Sophorae Tonkinensis Radix et Rhizoma, Sterculiae Lychnophorae Semen, Trichosanthis Radix, Ophiopogonis Radix, and Chebulae Fructus), is effective for clearing the throat and quenching thirst, treating aphasia and hoarseness, and relieving sore throat, dry mouth, and dry tongue [1].

(a)

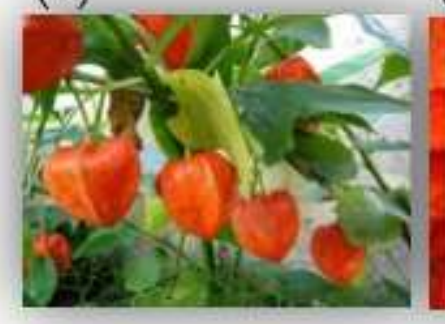

(b)

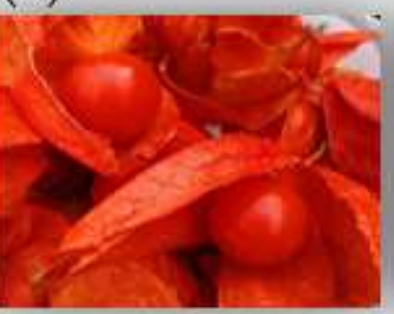

(c)

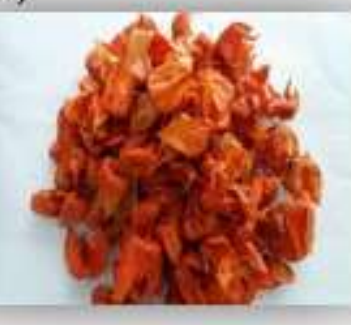

(d)

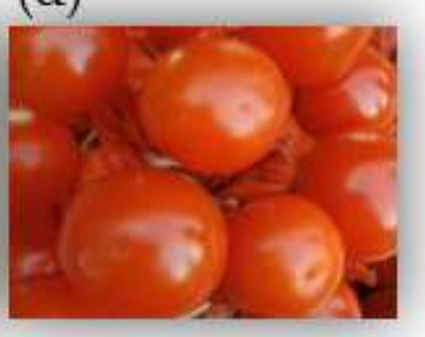

Figure 1. Images of P. alkekengi. (a) The whole plant; (b) Calyxes and fruits; (c) Calyxes; (d) Fruits.

In the last decades, reviews concerning research progress on the calyxes and fruits of P. alkekengi have been published, mainly focusing on the chemical components, traditional uses, toxicology, and pharmacological activities [6]; however, thus far, there are no reports on structural analysis, quality control, and pharmacokinetics. In recent years, new pharmacological activities have been discovered, and the main active ingredients in $P$. alkekengi are physalins and flavonoids [7]. Therefore, we herein provide a literature review on the structural analysis of physalins and flavonoids in the calyxes and fruits of $P$. alkekengi. We have also prepared a comprehensive and up-to-date report for the known pharmacological activities. In addition, the quality control and pharmacokinetics studies are summarized in detail. We hope that the current review will provide a theoretical basis and valuable data for future in-depth studies and the development of useful applications.

\section{Structural Analysis}

\subsection{Physalins}

P. alkekengi, a high-value multipurpose medicinal plant, is a rich reservoir of structurally diverse and biologically active terpenoids, termed physalins. Thus far, $>70$ physalin-type natural products have been isolated; most of them possess a 13,14-seco-16,24-cycloergostane skeleton, with anolides with a C-22, C-26 $\delta$-lactone side chain or C-23, C-26 $\gamma$-lactone side chain of C28 ergostane-type steroids [8,9]. According to the bonding type of C-14, physalins can be divided into two subtypes: physalins (Type I), in which C-14 is connected to C-17 through oxygen to form an acetal bridge, and neophysalins (Type II), where C-14 is connected to C-16 and C-15/C-17 is esterified to form lactone [10].

As shown in Figure 2, the synthesis of physalins can be divided into three steps. In step 1,5-carbon precursor isopentenyl diphosphate and dimethylallyl pyrophosphate are synthesized via cytosolic mevalonate (MEV) and plastid localized 2-C-methyl-D-erythritol4-phosphate (MEP) pathways, respectively; this is the first step toward the synthesis of physalins in plants [11]. In addition, 1-deoxy-D-xylulose-5-phosphate reductase and 3-hydroxy-3-methylglutaryl-coenzyme A reductase are key enzymes that regulate the 
MEP and MEV pathways, respectively [12]. In step 2, farnesyl pyrophosphate synthase catalyzes the conversion of isopentenyl diphosphate and dimethylallyl diphosphate to farnesyl pyrophosphate [13]. Additionally, farnesyl pyrophosphate is converted to 24-methylene cholesterol under the action of enzymes (squalene synthase, squalene epoxidase, cycloartenol synthase, etc.). It was also confirmed that cycloartenol, cycloeucalenol, and obtusifoliol are primary intermediates in the synthesis of the 24-methylene cholesterol $[14,15]$. In step 3, the skeletons of physalins (Types I and II) were produced from 24-methylene cholesterol $[13,16,17]$. Furthermore, the racemic DEFGH-ring moiety of physalins (Type I) is synthesized through enzymatic kinetic resolution [18]. The intermediate physalins were synthesized by domino ring transformation, a reoptimization of the 2,3-wittig rearrangement, and methylation steps. The DEFGH ring moiety was synthesized from precursors 2-methoxy-5-methylcyclohexa-2,5-diene-1,4-dione and (E)-1-((buta-1, 3-dien-1-yloxy)methyl)-4-methoxybenzene and an intermediate of a-allylic alcohol $[19,20]$. Additionally, physalins can be converted to neophysalins through an acid-induced benzilic acid-type rearrangement reaction [10].

The skeletons of physalins, through various biochemical reactions (i.e., desaturation, methylation, hydroxylation, epoxidation, cyclization, chain elongation, and glycosylation), lead to the production of various physalins [21]. As shown in Figure 3, Wu et al. [10] proposed the plausible biogenetic pathway for physalin IX, physalin $\mathrm{V}$, aromaphysalin $\mathrm{B}$, and physalinol A. For example, the epoxidation and hydroxylation of physalin B could produce an intermediate, which could be further ring-cleaved between C-1 and C-10, subjected to lactonization, and dehydrated to yield physalin V. Meanwhile, the cyclization of physalin $B$ between C- 11 and C-15 afforded the intermediate; subsequently, the intermediate was further epoxidized and hydrated to obtain physalin IX.

\subsection{Flavonoids}

Flavonoids are the second major component of P. alkekengi, with a common C6-C3$\mathrm{C} 6$ tricyclic skeleton $[22,23]$. The main flavonoid synthetic pathway has been characterized in P. alkekengi (Figure 4). The C6-C3-C6 carbon backbone was first synthesized through the phenylpropanoid pathway, transforming phenylalanine into 4-coumaroylcoenzyme A, which finally enters the flavonoid synthesis pathway [24,25]. Next, 4-coumaroyl -coenzyme A combines with three molecules of malonyl-coenzyme A to yield naringenin, which is the source of all flavonoids. Chalcone synthase and chalcone isomerase are the enzymes involved in the two-step condensation [26-28]. Naringenin is subsequently converted to luteolin through two reactions catalyzed by flavanone 3 '-hydroxylase and flavonol synthase. At the same time, the conversion of naringenin by flavanone 3hydroxylase yields dihydrokaempferol that can be hydroxylated on the 3' position of the B-ring by flavanone 3 '-hydroxylase, thereby producing dihydroquercetin. The subsequent steps of dihydrokaempferol and dihydroquercetin produce kaempferol and quercetin by flavonol synthase, respectively. The last step of quercetin for the formation of stable compounds involves glycosylation by the enzyme uridine diphosphate-glucose: flavonoid-3-Oglucosyltransferase. Finally, quercetin is further converted to quercetin-3-glucoside [29,30]. 


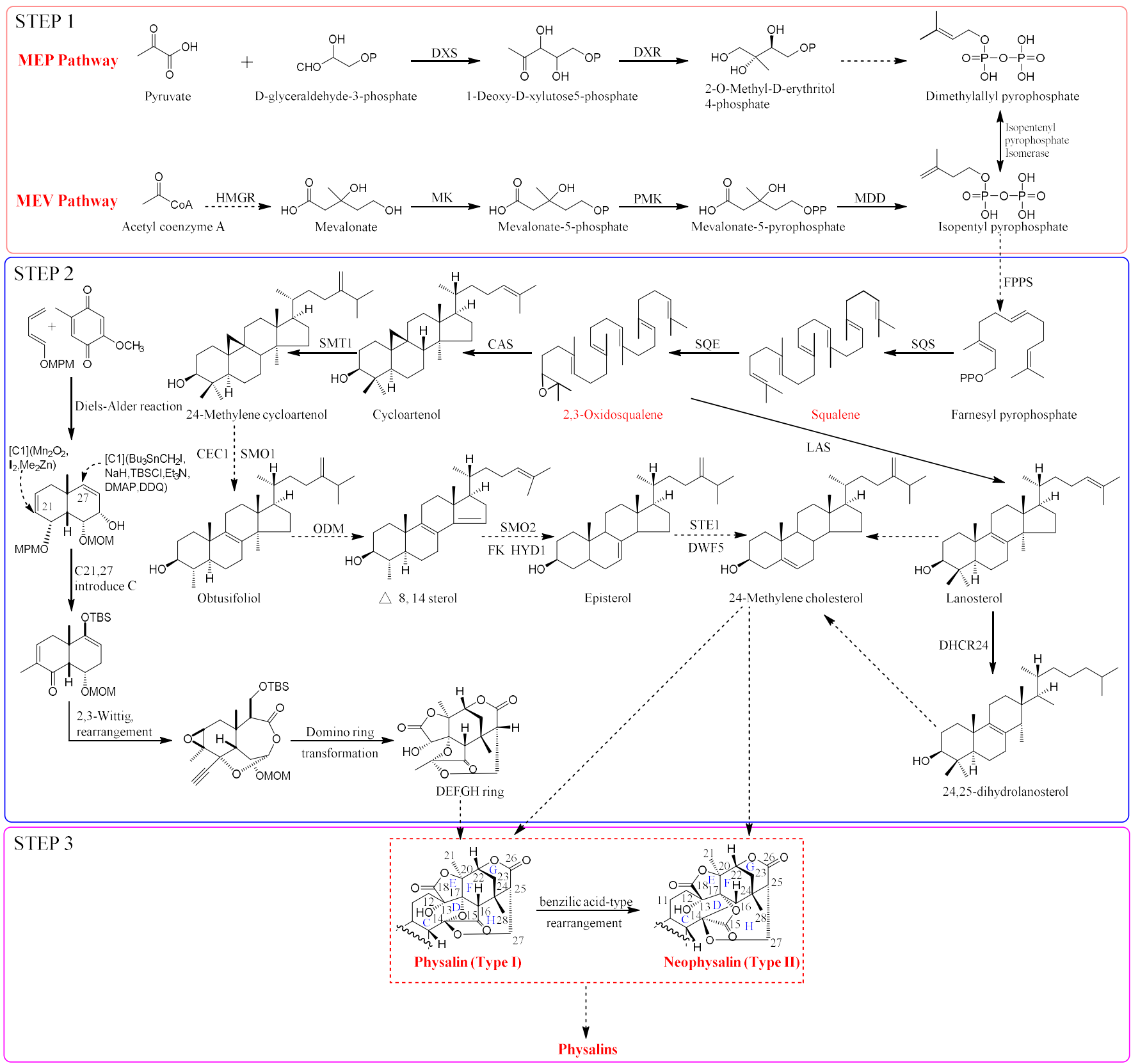

Figure 2. An overview of physalin synthesis in the calyxes and fruits of P. alkekengi. Solid oneheaded arrows indicate single-step irreversible reactions, while dotted arrows indicate several steps of reactions. Abbreviations: Bu, butyl; CAS, cycloartenol synthase; CEC1, cycloeucalenol cycloisomerase; CoA, coenzyme A; DDQ, 2,3-dichloro-5,6-dicyano-1,4-benzo-quinone; DHCR24, 24-dehydrocholesterol reductase; DMAP, 4-dimethylaminopyridine; DWF5, sterol delta-7 reductase; DXR, 1-deoxy-D-xylulose-5-phosphate reductase; DXS, 1-deoxy-D-xylulose-5-phosphate synthase; Et, C2H5; FK, delta 14-sterol reductase; FPPS, farnesyl diphosphate synthase; HMGR, 3-hydroxy3-methylglutaryl-coenzyme A reductase; HYD1, C-7,8 sterol isomerase; LAS, lanosterol synthase; MDD, mevalonate diphosphosphate decarboxylase; Me, CH3; MEP, 2-C-methyl-D-erythritol4-phosphate; MEV, mevalonate; MK, mevalonate kinase; MOM, methoxymethyl; MPM, paramethoxyphenylmethyl; ODM, obtusifoliol-14-demethylase; PMK, phosphomevalonate kinase; SMO1, sterol- $4 \alpha$-methyl oxidase 1 ; SMO2, sterol- $4 \alpha$-methyl oxidase 2; SMT1, sterol methyl transferase 1; SQS, squalene synthase; SQE, squalene epoxidase; STE1, C-5 sterol desaturase; TBS, tert-butyldimethylsilyl. 


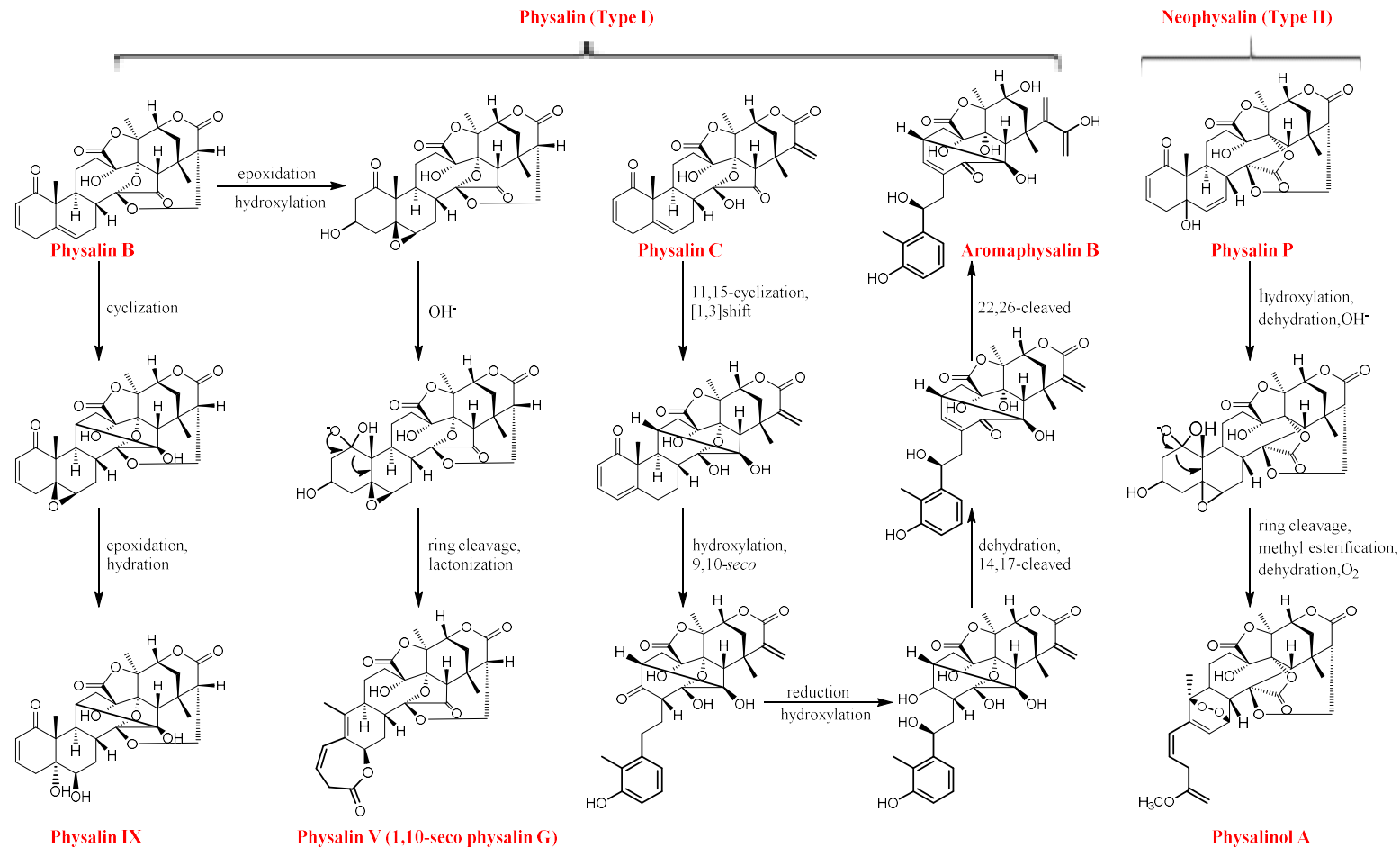

Figure 3. Biogenetic pathway of physalins in the calyxes and fruits of P. alkekengi.
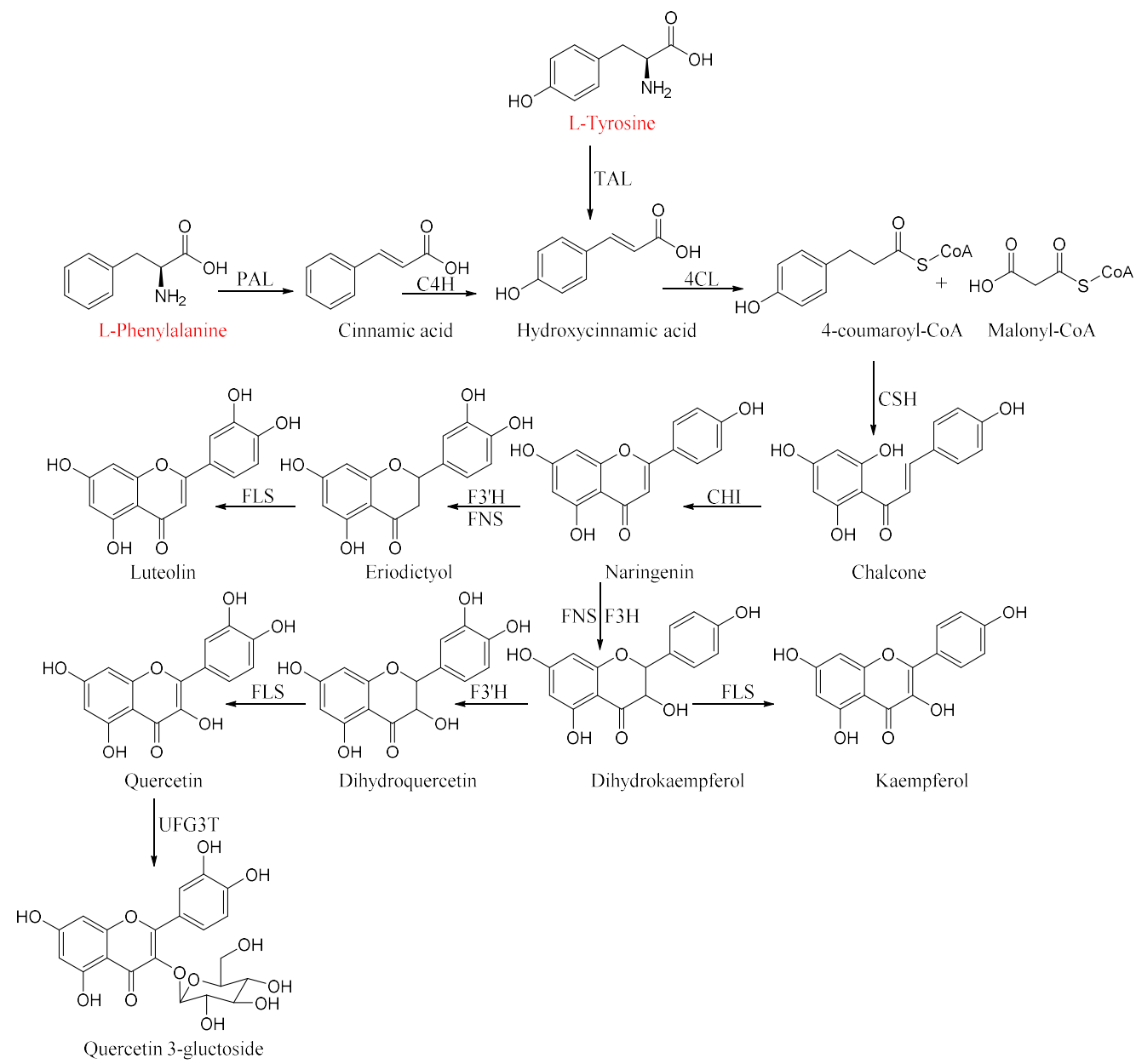

Figure 4. An overview of flavonoid synthesis in the calyxes and fruits of P. alkekengi. Abbreviations: 
4CL, 4-coumaryl-CoA ligase; $\mathrm{C} 4 \mathrm{H}$, cinnamate-4-hydroxylase; $\mathrm{CHI}$, chalcone isomerase; $\mathrm{CHS}$, chalcone synthase; CoA, coenzyme A; PAL, phenylalnine ammonialyase; F3'H, flavanone 3'-hydroxylase; FLS, flavonol synthase; FNS, flavone synthase; TAL, tyrosine ammonialyase; UFG3T, uridine diphosphateglucose: flavonoid-3-O-glucosyltransferase.

\section{Quality Control}

The quality control of calyxes and fruits is extremely important for their use. Extensive studies evaluated methods for the analysis of calyxes and fruits. According to the Chinese Pharmacopoeia, and based on the morphological, microscopic, and high-performance liquid chromatography (HPLC) analysis and thin-layer chromatography (TLC) identification, the minimum content of luteoloside for qualifying the calyxes and fruits of P. alkekengi is $\geq 0.10 \%$ [1]. However, due to the complex chemical components and diverse pharmacological activities of herbal medicines, a single quantitative marker appears to be insufficient for the assessment of quality. Currently, multiple compounds (mainly physalins, flavonoids, and polysaccharides) have been used to validate the quality of this herb by TLC, HPLC, and ultra-performance liquid chromatography-mass spectrometry (UPLC/MS) [31,32].

To obtain reliable pharmacological effects, the concentration of the chemical components of calyxes and fruits should be controlled; it is mainly determined by the season and harvesting time, as well as climatic and geographical conditions [33,34]. The effective composition of calyxes significantly changes with the growth period during harvest. A study showed that the content of physalin D in immature calyxes $(0.7880 \pm 0.0612 \%)$ was four-fold higher than that measured in mature calyxes $(0.2028 \pm 0.016 \%)$. Of note, the content of physalin D in fruits was markedly lower (immature fruits: $0.0992 \pm 0.0083 \%$; mature fruits: $0.0259 \pm 0.0021 \%$ ) [35]. Kranjc et al. [36] developed the first high-performance thin-layer chromatography (HPTLC) and HPTLC-MS/MS methods that can characterize physalins in crude extracts obtained from different parts of $P$. alkekengi at different stages of maturity. These findings indicated that only certain parts of the plant are appropriate for specific pharmaceutical applications. The HPTLC method overcame some of the drawbacks in analytical physalin profiling, providing a new approach to quality control for $P$. alkekengi. In addition, 31 samples of P. alkekengi collected from different habitats were analyzed using fingerprinting. The results showed that the contents of Baishan, Xinxiang, and Shenyang differed considerably [37]. Huang et al. [38] reported the fragmentation behavior of major physalins in P. alkekengi calyxes via ultra-high performance liquid chromatography (UHPLC)-quadrupole time of flight tandem mass spectrometry (QTOFMS/MS). The content of 4,7-didehydroneophysalin B in fruits and calyxes of P. alkekengi was determined by HPLC and UPLC-MS/MS. The results showed that the contents of 4,7-didehydroneophysalin B were $2.18 \%$ (50\% ethanol extract), $0.42 \%$ (70\% ethanol extract) by HPLC, and 15.75-70.88 $\mu \mathrm{g} / \mathrm{g}$ by UPLC-MS/MS. The results suggested that the content of 4,7-didehydroneophysalin B is relatively high and could be used as an index for the quality control of the medicinal material [39-41]. The contents of luteoloside, polysaccharides, reducing sugar, lutein, and $\beta$-carotene in samples obtained from different habitats were compared, revealing significant differences [42-44]. Moreover, some researchers have determined the contents of luteolin and luteoloside in pharmaceutical preparations (i.e., Physalis permviana liquid and Jinhuang yanyan tablets) $[45,46]$. These data provided an important theoretical basis for the harvesting of P. alkekengi, identification of physalins, and evaluation of the clinical applications of this medicinal herb. However, wide variations were observed in the contents of these compounds due to differences in the sources and time points of sample collection. Furthermore, the fruits of P. alkekengi also contained organic acids and smaller amounts of (hydroxy)cinnamoyl hexosides and amino acids [5]. The quantitative analysis of P. alkekengi is shown in Table 1. 
Table 1. Quantitative analysis for the quality control of P. alkekengi.

\begin{tabular}{|c|c|c|c|c|}
\hline Analytes & Method & Part Used & Results & Reference \\
\hline Physalins A, O, L, and B & HPLC & Fruits and calyxes & $\begin{array}{l}\text { In } 10 \text { habitats: } 1.04-3.12, \\
0.99-2.66,0.59-0.91, \text { and } \\
0.54-1.31 \mathrm{mg} / \mathrm{g}, \text { respectively. }\end{array}$ & [32] \\
\hline $\begin{array}{l}\text { Physalins B, D, G, and H, } \\
\text { 4,7-didehydroneophysalin B }\end{array}$ & UPLC-MS/MS & $\begin{array}{l}\text { Fruits and calyxes } \\
\text { Calyxes }\end{array}$ & $\begin{array}{l}\text { In } 14 \text { habitats: } 30.75-749.13 \text {, } \\
59.63-1046.63,15.25-527.15, \\
1.00-254.05,15.75-70.88 \mu \mathrm{g} / \mathrm{g} \text {, } \\
\text { respectively } \\
467.84,560.34,352.06,156.69, \\
43.22 \mu \mathrm{g} / \mathrm{g}, \text { respectively }\end{array}$ & [39] \\
\hline
\end{tabular}

\begin{tabular}{|c|c|}
\hline Physalin D & RP-HPLC-UV \\
\hline
\end{tabular}

In mature and immature:

$0.2028 \pm 0.0160 \%$,

$0.7880 \pm 0.0612 \%$, respectively

In mature and immature:

$0.0259 \pm 0.0021 \%$

$0.0992 \pm 0.0083 \%$, respectively

\begin{tabular}{|c|c|c|c|c|}
\hline 4,7-didehydroneophysalin B & HPLC & Fruits & $0.02 \%$ & [40] \\
\hline 4,7-didehydroneophysalin B & HPLC & Fruits and calyxes & $\begin{array}{l}50 \% \text { and } 70 \% \text { ethanol extract: } \\
2.18 \%, 0.42 \% \text {, respectively }\end{array}$ & [41] \\
\hline
\end{tabular}

\begin{tabular}{lll}
\hline & & In 6 habitats: $0.048-0.24$, \\
Physalins A, P and O, & Fruits & $0.04-0.2,0.36-1.8,0.052-0.26$, \\
& & \\
Luteoloside, luteolin & & $0.04-0.2 \mu \mathrm{g} / \mathrm{mL}$, respectively
\end{tabular}

\begin{tabular}{lllll}
\hline Luteoloside & TLC & Fruits and calyxes & In 11 habitats: $0.11-2.27 \mathrm{mg} / \mathrm{g}$ & [42] \\
\hline Luteolin & HPLC & $\begin{array}{l}\text { Physalis permviana } \\
\text { liquid }\end{array}$ & $0.75 \mu \mathrm{g} / \mathrm{mL}$ & {$[45]$}
\end{tabular}

Polysaccharides

Reducing sugar

$\begin{array}{ll}\text { UV } & \text { Calyxes } \\ \text { Fruits }\end{array}$

In 52 habitats: 0.34-9.67,

$1.32-146.53 \mathrm{mg} / \mathrm{g}$, respectively

In 50 habitats: $2.47-11.82$,

$181.97-321.57 \mathrm{mg} / \mathrm{g}$,

respectively

\begin{tabular}{|c|c|c|c|c|}
\hline $\begin{array}{l}\text { Luteoloside } \\
\text { Luteolin }\end{array}$ & HPLC & $\begin{array}{l}\text { Jinhuang yanyan } \\
\text { tablets }\end{array}$ & $\begin{array}{l}0.14 \%-0.15 \%, \\
0.0066 \%-0.0070 \% \text {, respectively }\end{array}$ & [46] \\
\hline $\begin{array}{l}\text { Lutein } \\
\beta \text {-carotene }\end{array}$ & HPLC-DAD-APCI-MS & Fruits & $\begin{array}{l}19.8-21.6 \mathrm{mg} / 100 \mathrm{~g} \text { of total } \\
\text { lutein and } \beta \text {-carotene contents }\end{array}$ & [44] \\
\hline $\begin{array}{l}\text { Citric acid } \\
\text { Malic acid } \\
\text { Tartaric acid } \\
\text { Ascorbic acid }\end{array}$ & HPLC-UV & Fruits & $\begin{array}{l}903-920 \mathrm{mg} / 100 \mathrm{~g} \\
396-554 \mathrm{mg} / 100 \mathrm{~g} \\
261-325 \mathrm{mg} / 100 \mathrm{~g} \\
26-32 \mathrm{mg} / 100 \mathrm{~g}\end{array}$ & [5] \\
\hline $\begin{array}{l}\text { (hydroxy)cinnamoyl } \\
\text { hexosides } \\
\text { Sinapoyl } \\
\text { Feruloyl hexosides }\end{array}$ & HPLC-DAD-ESI-MS & Fruits & $\begin{array}{l}70.8-81.6 \mathrm{mg} / \mathrm{kg} \\
57.8-68.0 \mathrm{mg} / \mathrm{kg} \\
10.6-13.6 \mathrm{mg} / \mathrm{kg}\end{array}$ & [5] \\
\hline $\begin{array}{l}\text { Aromatic amino acids and } \\
\text { amino derivatives }\end{array}$ & HPLC-DAD-ESI-MS & Fruits & $50.9-63.5 \mathrm{mg} / \mathrm{kg}$ & [5] \\
\hline
\end{tabular}

Abbreviations: APCI, atmospheric pressure chemical ionization; DAD, diode array detection; ESI, electrospray ionization interface; HPLC, high-performance liquid chromatography; MS, mass spectrometry; MS/MS, tandem mass spectrometry; RP, reverse phase; TLC, thin-layer chromatography; UPLC, ultra-performance liquid chromatography; UV, ultraviolet.

\section{Pharmacology}

Pharmacological experiments showed that the various crude extracts and compounds isolated from P. alkekengi have diverse biological activities (e.g., anti-inflammatory, antitumor, immunosuppressive, anti-microbial, anti-leishmanial, anti-asthmatic, anti-diabetic, 
etc.). In addition, the mechanisms of action of the anti-inflammatory and anti-tumor activities were also reported. The main pharmacological activities of crude extracts and compounds are shown in Table 2.

Table 2. Pharmacological effects of P. alkekengi.

\begin{tabular}{|c|c|c|c|c|c|}
\hline $\begin{array}{l}\text { Pharmacological } \\
\text { Activity }\end{array}$ & Animal/Cell Models & Constituent/Extract & Detail & Dosage & Reference \\
\hline \multirow[t]{18}{*}{$\begin{array}{l}\text { Anti-inflammatory } \\
\text { activity }\end{array}$} & LPS-induced 264.7 cells & $\begin{array}{l}\text { Physalins A, O, L, G } \\
\text { Isophysalin A }\end{array}$ & Induced NO production & $20 \mu \mathrm{M}$ & [47] \\
\hline & $\begin{array}{l}\text { IFN- } \gamma \text {-stimulated } \\
\text { macrophages } \\
\text { LPS-stimulated } \\
\text { macrophages }\end{array}$ & Physalins B, F, G & $\begin{array}{l}\text { Reduced NO production; inhibited TNF- } \alpha \text {, } \\
\text { IL-6, IL-12 }\end{array}$ & $2 \mu \mathrm{g} / \mathrm{mL}$ & [48] \\
\hline & C57BL/6 mice & Physalins B, F & $\begin{array}{l}\text { Suppressed the increase in TNF- } \alpha \text {; } \\
\text { increased vascular permeability; prevented } \\
\text { neutrophil influx }\end{array}$ & $20 \mathrm{mg} / \mathrm{kg}$ & [49] \\
\hline & LPS-induced 264.7 cells & Physalin B & Decreased the levels of TNF- $\alpha$, IL- 6 , IL- $1 \beta$ & $0.25,0.5,1.0 \mu \mathrm{M}$ & [50] \\
\hline & $\begin{array}{l}\text { LPS/IFN- } \gamma \text {-induced } \\
\text { macrophages } \\
\text { IL-4/IL-13-induced } \\
\text { macrophages } \\
\text { LPS-induced } \\
\text { C57BL/ } 6 \text { mice }\end{array}$ & Physalin D & $\begin{array}{l}\text { In vitro: activated signal transducer and } \\
\text { activator of STAT6 pathway; suppressed } \\
\text { STAT1 activation; blocked STAT1 nuclear } \\
\text { translocation } \\
\text { In vivo: reduced inducible iNOS cell } \\
\text { number; increased CD206+ cell number }\end{array}$ & $5 \mu \mathrm{M}$ & [51] \\
\hline & $\begin{array}{l}\text { LPS-stimulated RAW } \\
264.7 \text { cells }\end{array}$ & Physalin E & $\begin{array}{l}\text { Inhibited the generation of TNF- } \alpha \text {, IL- } 6 \text {, } \\
\text { NF- } \kappa \text { B p } 65 \text {; reduced the degradation of } \\
\text { I-kappa B protein }\end{array}$ & $12.5,25,50 \mu \mathrm{M}$ & [52] \\
\hline & $\begin{array}{l}\text { TPA-induced acute ear } \\
\text { edema in mice } \\
\text { Oxazolone-induced } \\
\text { chronic dermatitis in } \\
\text { mice }\end{array}$ & Physalin E & $\begin{array}{l}\text { Reduced ear edema response and } \\
\text { myeloperoxidase activity; suppressed } \\
\text { increase in ear thickness and levels of } \\
\text { TNF- } \alpha \text { and IFN- } \gamma\end{array}$ & $0.125,0.25,0.5 \mathrm{mg} / \mathrm{ear}$ & {$[53]$} \\
\hline & DBA/1 mice & Physalin F & $\begin{array}{l}\text { Decreased paw edema and joint } \\
\text { inflammation }\end{array}$ & $60 \mathrm{mg} / \mathrm{kg}$ & {$[54]$} \\
\hline & $\begin{array}{l}\text { LPS-induced } \\
\text { macrophages }\end{array}$ & $\begin{array}{l}\text { Physalin X } \\
\text { Aromaphysalin B }\end{array}$ & Inhibited NO production & $\begin{array}{l}\mathrm{IC}_{50}=68.50,29.69 \mu \mathrm{M}, \\
\text { respectively }\end{array}$ & [55] \\
\hline & $\begin{array}{l}\text { LPS-induced } \\
\text { macrophages }\end{array}$ & $\begin{array}{l}\text { Physalins B, F, H, V, D1, } \\
\text { VII, I } \\
\text { Isophysalin B }\end{array}$ & Inhibited NO production & $\begin{array}{l}\mathrm{IC}_{50}=0.32-4.03 \\
12.83-34.19 \mu \mathrm{M}, \\
\text { respectively. }\end{array}$ & [56] \\
\hline & $\begin{array}{l}\text { LPS-induced } \\
\text { macrophages }\end{array}$ & $\begin{array}{l}\text { Physalins A, B, F } \\
\text { Ombuine } \\
\text { Luteolin }\end{array}$ & Inhibited NO production & $\begin{array}{l}\mathrm{IC}_{50}=2.57 \pm 1.18 \\
0.84 \pm 0.64,0.33 \pm 0.17 \\
2.23 \pm 0.19 \\
7.39 \pm 2.18 \mu \mathrm{M} \\
\text { respectively. }\end{array}$ & {$[57]$} \\
\hline & $\begin{array}{l}\text { LPS } / \text { IFN- } \gamma \text {-stimulated } \\
\text { macrophages } \\
\text { ICR mice }\end{array}$ & Luteolin & $\begin{array}{l}\text { In vitro: suppressed the production of IL-6, } \\
\text { IL-12, and TNF- } \alpha \\
\text { In vivo: inhibited paw edema }\end{array}$ & $\begin{array}{l}20 \mu \mathrm{M} \\
20 \mathrm{mg} / \mathrm{kg}\end{array}$ & [58] \\
\hline & KF-8 cells & $\begin{array}{l}\text { Apigenin } \\
\text { Lutelin }\end{array}$ & $\begin{array}{l}\text { Inhibited NF- } \kappa \text { B activation and the } \\
\text { expression of CCL2/MCP-1 and } \\
\text { CXCL1/KC }\end{array}$ & $20 \mu \mathrm{M}$ & [59] \\
\hline & $\begin{array}{l}\text { LPS-induced } \\
\text { macrophages }\end{array}$ & $\begin{array}{l}\text { Kaempferol } \\
\text { Quercetin }\end{array}$ & $\begin{array}{l}\text { Inhibited STAT- } 1 \text { and NF- } \kappa \text { B activation, } \\
\text { iNOS protein and mRNA expression, and } \\
\text { NO production }\end{array}$ & $100 \mu \mathrm{M}$ & {$[60,61]$} \\
\hline & $\begin{array}{l}\text { LPS-stimulated THP-1 } \\
\text { cells } \\
\text { ICR mice }\end{array}$ & $70 \%$ ethanol extract & $\begin{array}{l}\text { In vitro: reduced the production of NO, } \\
\text { PGE2, TNF- } \alpha \text {, IL-1, iNOS, and COX-2 } \\
\text { In vivo: reduced ear edema; induced } \\
\text { granulomatous tissue formation }\end{array}$ & $500 \mu \mathrm{g} / \mathrm{mL}$ & [62] \\
\hline & Wistar rats & Methanol extract & Reduced the paw volume & $500 \mathrm{mg} / \mathrm{kg}$ & {$[63]$} \\
\hline & $\begin{array}{l}\text { LPS-induced } \\
\text { macrophages }\end{array}$ & Physanosides B & Inhibited NO production & $\mathrm{IC}_{50}=9.93 \mu \mathrm{M}$ & [64] \\
\hline & $\begin{array}{l}\text { LPS-induced } \\
\text { macrophages }\end{array}$ & $(6 \mathrm{~S}, 9 \mathrm{R})$-roseoside & Inhibited NO production & $\mathrm{IC}_{50}=7.31 \mu \mathrm{M}$ & {$[65]$} \\
\hline \multirow[t]{3}{*}{ Anti-tumor activity } & HepG2 cells & Physalin A & $\begin{array}{l}\text { Activated the Nrf2-ARE pathway and its } \\
\text { target genes }\end{array}$ & $20 \mu \mathrm{M}$ & [65] \\
\hline & $\begin{array}{l}\text { Non-small cell lung } \\
\text { cancer } \\
\text { BALB / c mice }\end{array}$ & Physalin A & $\begin{array}{l}\text { In vitro: suppressed both constitutive and } \\
\text { induced STAT3 activity } \\
\text { In vivo: suppressed tumor xenograft } \\
\text { growth }\end{array}$ & $\begin{array}{l}5,10,15 \mu \mathrm{M} \\
40,80 \mathrm{mg} / \mathrm{kg}\end{array}$ & [66] \\
\hline & $\begin{array}{l}\text { Human melanoma } \\
\text { A375-S2 cells }\end{array}$ & Physalin A & $\begin{array}{l}\text { Activated transmembrane death receptor; } \\
\text { Induced poptosis via apoptotic (intrinsic } \\
\text { and extrinsic) pathway; up-regulated } \\
\text { p53-NOXA-mediated ROS generation }\end{array}$ & $15 \mu \mathrm{M}$ & [67] \\
\hline
\end{tabular}


Table 2. Cont.

\begin{tabular}{|c|c|c|c|c|c|}
\hline $\begin{array}{l}\text { Pharmacological } \\
\text { Activity }\end{array}$ & Animal/Cell Models & Constituent/Extract & Detail & Dosage & Reference \\
\hline & $\begin{array}{l}\text { Human HT1080 } \\
\text { fibrosarcoma cells }\end{array}$ & Physalin A & Upregulated CASP3, CASP8 expression & $\mathrm{IC}_{50}=10.7 \pm 0.91 \mu \mathrm{M}$ & [68] \\
\hline & $\begin{array}{l}\text { Human melanoma } \\
\text { A375-S2 cells }\end{array}$ & Physalin A & $\begin{array}{l}\text { Repressed the production of RNS and ROS; } \\
\text { triggered the expression of iNOS and NO }\end{array}$ & $15 \mu \mathrm{M}$ & [69] \\
\hline & $\begin{array}{l}\text { Non-small cell lung } \\
\text { cancer }\end{array}$ & Physalin A & $\begin{array}{l}\text { Induced G2/M cell cycle arrest; increased } \\
\text { the amount of intracellular ROS }\end{array}$ & $\mathrm{IC}_{50}=28.4 \mu \mathrm{M}$ & {$[70]$} \\
\hline & $\begin{array}{l}\text { Prostate cancer cells } \\
\text { (CWR22Rv1, C42B) }\end{array}$ & Physalins A, B & $\begin{array}{l}\text { Inhibited the growth of two cells; activated } \\
\text { the JNK and ERK pathway }\end{array}$ & $\begin{array}{l}\mathrm{IC}_{50}=14.2,9.6 \mu \mathrm{M}, \\
\text { respectively }\end{array}$ & {$[71]$} \\
\hline & $\begin{array}{l}\text { Non-small cell lung } \\
\text { cancer }\end{array}$ & Physalin B & $\begin{array}{l}\text { Exhibited anti-proliferative and apoptotic } \\
\text { activity; downregulated the } \\
\text { CDK1/CCNB1 complex; upregulated p21 }\end{array}$ & $5,10,20 \mu \mathrm{mol} / \mathrm{L}$ & {$[72]$} \\
\hline & $\begin{array}{l}\text { Human melanoma } \\
\text { A375 cells }\end{array}$ & Physalin B & $\begin{array}{l}\text { Activated the expression of the NOXA, } \\
\text { BCL2 associated X (Bax), and CASP3 }\end{array}$ & $3 \mu \mathrm{g} / \mathrm{mL}$ & [73] \\
\hline & $\begin{array}{l}\text { Human HCT116 colon } \\
\text { cancer cells }\end{array}$ & Physalin B & $\begin{array}{l}\text { Activated the ERK, JNK, and p38 MAPK } \\
\text { pathways; increased ROS generation }\end{array}$ & $\mathrm{IC}_{50}=1.35 \mu \mathrm{mol} / \mathrm{L}$ & {$[74]$} \\
\hline & $\begin{array}{l}\text { Human DLD-1 colon } \\
\text { cancer cells }\end{array}$ & Physalin B & $\begin{array}{l}\text { Inhibited TNF } \alpha \text {-induced NF- } \kappa \text { B activation; } \\
\text { induced the proapoptotic protein NOXA } \\
\text { generation }\end{array}$ & $5 \mu \mathrm{M}$ & [75] \\
\hline & $\begin{array}{l}\text { Breast cancer cells } \\
\text { (MCF-7, MDA-MB-231, } \\
\text { T-47D) }\end{array}$ & Physalin B & $\begin{array}{l}\text { Induced cell cycle arrest at G2/M phase; } \\
\text { promoted the cleavage of PARP, CASP3, } \\
\text { CASP7, and CASP9; inactivated Akt and } \\
\text { P13K phosphorylation }\end{array}$ & $2.5,5,10 \mu \mathrm{M}$ & [76] \\
\hline & $\begin{array}{l}\text { TNF- } \alpha \text {-stimulated } \\
\text { HeLa cells }\end{array}$ & Physalins B, C, F & $\begin{array}{l}\text { Inhibited the phosphorylation and } \\
\text { degradation of } \mathrm{I} \kappa \mathrm{B} \alpha \text { and NF- } \kappa B \text { activation }\end{array}$ & $\begin{array}{l}\mathrm{IC}_{50}=6.07,6.54 \\
2.53 \mu \mathrm{M}, \text { respectively }\end{array}$ & [9] \\
\hline & $\begin{array}{l}\text { Tumor cells (A549, } \\
\text { K562) }\end{array}$ & $\begin{array}{l}(17 \mathrm{~S}, 20 \mathrm{R}, 22 \mathrm{R})-5 \beta, 6 \beta- \\
\text { epoxy-18,20-dihydroxy- } \\
\text { 1-ox- } \\
\text { owitha-2,24-dienolide } \\
\text { withaphysalin B }\end{array}$ & $\begin{array}{l}\text { Suppressed the PI3K/Akt/mTOR } \\
\text { signaling pathway }\end{array}$ & $\mathrm{IC}_{50}=1.9-4.3 \mu \mathrm{M}$ & [77] \\
\hline & $\begin{array}{l}\text { Tumor cells (B-16, } \\
\text { HCT-8, PC3, } \\
\text { MDA-MB-435, } \\
\text { MDA-MB-231, MCF-7, } \\
\text { K562, CEM, HL-60) } \\
\text { Swiss mice }\end{array}$ & Physalins B, D & $\begin{array}{l}\text { In vitro: displayed activity against several } \\
\text { cancer cell lines } \\
\text { In vivo: inhibited the proliferation of cells; } \\
\text { reduced Ki67 staining }\end{array}$ & $\begin{array}{l}0.58-15.18, \\
0.28-2.43 \mu \mathrm{g} / \mathrm{mL} \\
\text { respectively } \\
10,25 \mathrm{mg} / \mathrm{kg}\end{array}$ & [78] \\
\hline & $\begin{array}{l}\text { Human cancer cells } \\
\text { (C4-2B, 22Rv1, 786-O, } \\
\text { A-498, ACHN, A375-S2) }\end{array}$ & Physalins B, F & Showed anti-proliferative activities & $\mathrm{IC}_{50}=0.24-3.17 \mu \mathrm{M}$ & [56] \\
\hline & $\begin{array}{l}\text { Human T cell leukemia } \\
\text { Jurkat cells }\end{array}$ & Physalins B, F & $\begin{array}{l}\text { Inhibited PMA-induced NF- } \kappa B \text { and } \\
\text { TNF- } \alpha \text {-induced NF- } \kappa B \text { activation }\end{array}$ & $8,16 \mu \mathrm{M}$, respectively & [79] \\
\hline & $\begin{array}{l}\text { HEK293T cells } \\
\text { BALB/c-nu/nu mice }\end{array}$ & Physalin F & $\begin{array}{l}\text { In vitro: decreased TOPFlash reporter } \\
\text { activity; promoted the proteasomal } \\
\text { degradation of } \beta \text {-catenin } \\
\text { In vivo: downregulated } \beta \text {-catenin }\end{array}$ & $\begin{array}{l}4 \mu \mathrm{M} \\
10,20 \mathrm{mg} / \mathrm{kg}\end{array}$ & {$[80]$} \\
\hline & T-47D cells & Physalin F & Activated the CASP3 and c-myc pathways & $\mathrm{IC}_{50}=3.60 \mu \mathrm{g} / \mathrm{mL}$ & [81] \\
\hline & $\begin{array}{l}\text { Human renal, } \\
\text { carcinoma cells (A498, } \\
\text { ACHN, UO-31) }\end{array}$ & Physalin F & $\begin{array}{l}\text { Induced cell apoptosis through the } \\
\text { ROS-mediated mitochondrial pathway; } \\
\text { suppressed NF- } \kappa \text { B activation }\end{array}$ & $1,3,10 \mu \mathrm{g} / \mathrm{mL}$ & [82] \\
\hline & PC-3 cancer cell lines & $\begin{array}{l}7 \beta \text {-ethoxyl-isophysalin } \\
C\end{array}$ & Showed apparent moderate activities & $\mathrm{IC}_{50}=8.26 \mu \mathrm{M}$ & [83] \\
\hline & $\begin{array}{l}\text { Human osteosarcoma } \\
\text { cells }\end{array}$ & Physakengose G & $\begin{array}{l}\text { Inhibited the epidermal growth factor } \\
\text { receptor/mTOR (EGFR/mTOR) pathway; } \\
\text { blocked autophagic flux through lysosome } \\
\text { dysfunction }\end{array}$ & $5,10,20 \mu \mathrm{M}$ & {$[84]$} \\
\hline \multirow[t]{3}{*}{$\begin{array}{l}\text { Immunosuppressive } \\
\text { activity }\end{array}$} & $\begin{array}{l}\text { Trypanosoma cruzi ( } T \text {. } \\
\text { cruzi)-infected insects }\end{array}$ & Physalin B & $\begin{array}{l}\text { Decreased number of } T \text {. cruzi Dm } 28 \mathrm{c} \text { and } T \text {. } \\
\text { cruzi transmission; inhibited the } \\
\text { development of parasites }\end{array}$ & $\begin{array}{l}1 \mathrm{mg} / \mathrm{mL} \\
20 \mathrm{ng} \\
57 \mathrm{ng} / \mathrm{cm}^{2}\end{array}$ & [85] \\
\hline & $\begin{array}{l}\text { H14 Trypanosoma } \\
\text { rangeli-infected } \\
\text { Rhodnius prolixus larvae }\end{array}$ & Physalin B & $\begin{array}{l}\text { Reduced the production of hemocyte } \\
\text { microaggregation and NO }\end{array}$ & $0.1,1 \mu \mathrm{g} / \mathrm{mL}$ & [86] \\
\hline & $\begin{array}{l}\text { T. cruzi trypomastigotes } \\
\text { BALB/c mice } \\
\text { macrophages }\end{array}$ & $\begin{array}{l}\text { Physalin B } \\
\text { Physalin F }\end{array}$ & $\begin{array}{l}\text { Displayed strongest effects against } \\
\text { epimastigote forms of T. cruzi }\end{array}$ & $\begin{array}{l}\mathrm{IC}_{50}=5.3 \pm 1.9, \\
5.8 \pm 1.5 \mu \mathrm{M}, \\
\text { respectively } \\
\mathrm{IC}_{50}=0.68 \pm 0.01 \\
0.84 \pm 0.04 \mu \mathrm{M}, \\
\text { respectively }\end{array}$ & [87] \\
\hline
\end{tabular}


Table 2. Cont.

\begin{tabular}{|c|c|c|c|c|c|}
\hline $\begin{array}{l}\text { Pharmacological } \\
\text { Activity }\end{array}$ & Animal/Cell Models & Constituent/Extract & Detail & Dosage & Reference \\
\hline & $\begin{array}{l}\text { Con A-induced spleen } \\
\text { cells } \\
\text { CBA mice }\end{array}$ & Physalins B, F, G & $\begin{array}{l}\text { In vitro: inhibited MLR and IL-2 } \\
\text { production } \\
\text { In vivo: prevented the rejection of } \\
\text { allogeneic heterotopic heart transplant }\end{array}$ & $\begin{array}{l}2 \mu \mathrm{g} / \mathrm{mL} \\
1 \mathrm{mg} / \mathrm{mouse} / \text { day }\end{array}$ & [88] \\
\hline & $\begin{array}{l}\text { Human T-cell } \\
\text { lymphotropic virus type } \\
1 \text { (HTLV-1)-infected } \\
\text { subjects }\end{array}$ & Physalin F & $\begin{array}{l}\text { Inhibited spontaneous proliferation; } \\
\text { reduced the levels of IL-2, IL-6, IL-10, } \\
\text { TNF- } \alpha \text {, and IFN- } \gamma\end{array}$ & $10 \mu \mathrm{M}$ & [89] \\
\hline & $\begin{array}{l}\text { T cells } \\
\text { BALB/c mice }\end{array}$ & Physalin $\mathrm{H}$ & $\begin{array}{l}\text { In vitro: suppressed proliferation and MLR } \\
\text { In vivo: inhibited delayed-type } \\
\text { hypersensitivity reactions and T-cell } \\
\text { response }\end{array}$ & $\begin{array}{l}\mathrm{IC}_{50}=0.69,0.39 \mu \mathrm{g} / \mathrm{mL} \\
\text { respectively } \\
\mathrm{IC}_{50}=2.75 \text { or } \\
3.61 \mu \mathrm{g} / \mathrm{mL}\end{array}$ & {$[90]$} \\
\hline & ICR mice & Polysaccharides & $\begin{array}{l}\text { Enhanced specific antibody titers } \\
\text { immunoglobulin G (IgG), IgG1, and IgG2b, } \\
\text { as well as the concentration of IL-2 and } \\
\text { IL-4 }\end{array}$ & $40 \mu \mathrm{g} /$ mice & [91] \\
\hline \multirow[t]{6}{*}{$\begin{array}{l}\text { Anti-microbial } \\
\text { activity }\end{array}$} & $\begin{array}{l}\text { Gram-positive bacteria: } \\
\text { Staphylococcus } \\
\text { epidermidis (S. } \\
\text { epidermidis), } \\
\text { Enterococcus faecalis (E. } \\
\text { faecalis), Staphylococcus } \\
\text { aureus (S. aureus), } \\
\text { Bacillus subtilis (B. } \\
\text { subtilis), Bacillus cereus } \\
\text { (B. cereus) }\end{array}$ & $\begin{array}{l}\text { Methanol extract } \\
\text { Dichloromethane } \\
\text { extract } \\
\text { Physalin D }\end{array}$ & Displayed moderate antibacterial activity & $\mathrm{MIC}=32-128 \mu \mathrm{g} / \mathrm{mL}$ & [92] \\
\hline & $\begin{array}{l}\text { Escherichia coli (E. coli), } \\
\text { B. subtilis }\end{array}$ & Physalins B, J, P & Showed high antibacterial activity & $\begin{array}{l}\text { MIC }=12.5-23.7, \\
23.23-24.34, \\
22.8-27.98 \mu \mathrm{g} / \mathrm{mL} \\
\text { respectively }\end{array}$ & [93] \\
\hline & $\begin{array}{l}\text { Mycobacterium } \\
\text { tuberculosis } \mathrm{H} 37 \mathrm{Rv}\end{array}$ & $\begin{array}{l}\text { Trichlormethane extract } \\
\text { Physalins B, D }\end{array}$ & Showed antibacterial activity & $\begin{array}{l}\mathrm{MIC}=32,>128 \\
32 \mu \mathrm{g} / \mathrm{mL}, \text { respectively }\end{array}$ & [94] \\
\hline & $\begin{array}{l}\text { Lactobacillus delbrueckii } \\
\text { (L. delbrueckii), } \\
\text { E. coli }\end{array}$ & $70 \%$ ethanol extract & $\begin{array}{l}\text { Promoted the growth of L. delbrueckii; } \\
\text { inhibited the growth of E. coli }\end{array}$ & $0.78-1.56 \mathrm{mg} / \mathrm{mL}$ & [95] \\
\hline & $\begin{array}{l}\text { Gram-positive bacteria: } \\
\text { S. aureus, S. epidermidis, } \\
\text { Staphylococcus } \\
\text { saprophyticus (S. } \\
\text { saprophyticus), } \\
\text { Enterococcus faecium (E. } \\
\text { faecium) } \\
\text { Gram-negative bacteria: } \\
\text { Pseudomonas aeruginosa } \\
\text { (P. aeruginosa), } \\
\text { Streptococcus pneumoniae } \\
\text { (S. pneumoniae), E. coli }\end{array}$ & $70 \%$ ethanol extract & Showed antibacterial activity & $\begin{array}{l}\mathrm{MIC}=0.825-1.65 \\
\mathrm{mg} / \mathrm{mL}\end{array}$ & [62] \\
\hline & $\begin{array}{l}\text { S. aureus, } B \text {. subtilis, } P \text {. } \\
\text { aeruginosa, E. coli }\end{array}$ & $\begin{array}{l}\text { Physakengoses B, E, F, } \\
\text { G, H, K, L, M, N, O }\end{array}$ & Showed potent inhibitory effects & $\mathrm{MIC}=2.16-14.9 \mu \mathrm{g} / \mathrm{mL}$ & {$[96,97]$} \\
\hline Anti-leishmanial & $\begin{array}{l}\text { Leishmania-infected } \\
\text { macrophages } \\
\text { Leishmania amazonensis } \\
\text {-infected BALB/c mice }\end{array}$ & Physalins B, F & $\begin{array}{l}\text { In vitro: reduced the percentage of } \\
\text { macrophages } \\
\text { In vivo: reduced the lesion size, the } \\
\text { parasite load, and histopathological } \\
\text { alterations }\end{array}$ & $\begin{array}{l}\mathrm{IC}_{50}=0.21 \text { and } 0.18 \mu \mathrm{M} \\
\text { respectively }\end{array}$ & [98] \\
\hline \multirow[t]{5}{*}{ Others } & Kunming mice & Water extract & $\begin{array}{l}\text { Decreased the expression of white blood } \\
\text { cells and eosinophils, IL-5, IFN- } \gamma \text {, Th1, } \\
\text { and Th2 }\end{array}$ & $0.25,5,1 \mathrm{~g} / \mathrm{mL}$ & [99] \\
\hline & $\begin{array}{l}\text { 3T3-L1 pre-adipocyte } \\
\text { cells } \\
\text { HepG2 cells } \\
\text { Male Sprague-Dawley } \\
\text { (SD) rats }\end{array}$ & Ethyl acetate extract & $\begin{array}{l}\text { In vitro: relieved oxidative stress; inhibited } \\
\alpha \text {-glucosidase activity. } \\
\text { In vivo: decreased FBG, TC, and TG }\end{array}$ & $300 \mathrm{mg} / \mathrm{kg}$ & [100] \\
\hline & Alloxan-induced mice & Polysaccharides & $\begin{array}{l}\text { Decreased FBG and GSP; increased FINS; } \\
\text { upregulated the PI3K, Akt, and GLUT4 } \\
\text { mRNA }\end{array}$ & $200,400,800 \mathrm{mg} / \mathrm{kg}$ & [101] \\
\hline & $\begin{array}{l}\text { High-fat diet-fed and } \\
\text { streptozotocin-induced } \\
\text { diabetic SD rats }\end{array}$ & Ethyl acetate extract & $\begin{array}{l}\text { Reduced the FBG, TC, TG, and GSP; } \\
\text { increased FINS }\end{array}$ & $300,600 \mathrm{mg} / \mathrm{kg}$ & [102] \\
\hline & $\begin{array}{l}\text { Wistar rats } \\
\text { Albino mice }\end{array}$ & $\begin{array}{l}\text { Aqueous methanolic } \\
\text { extract }\end{array}$ & $\begin{array}{l}\text { Reduced the intensity of gastric mucosal } \\
\text { damage; inhibited pain sensation }\end{array}$ & $\begin{array}{l}500 \mu \mathrm{g} / \mathrm{mL} \\
500 \mathrm{mg} / \mathrm{kg}\end{array}$ & [63] \\
\hline
\end{tabular}


Table 2. Cont.

\begin{tabular}{|c|c|c|c|c|c|}
\hline $\begin{array}{l}\text { Pharmacological } \\
\text { Activity }\end{array}$ & Animal/Cell Models & Constituent/Extract & Detail & Dosage & Reference \\
\hline & $\begin{array}{l}\text { LPS-induced acute lung } \\
\text { injury in BALB/c mice }\end{array}$ & $70 \%$ ethanol extract & $\begin{array}{l}\text { Reduced the release of TNF- } \alpha \text { and the } \\
\text { accumulation of oxidation products; } \\
\text { decreased the levels of NF- } \mathrm{KB} \text {, } \\
\text { phosphorylated-p38, ERK, JNK, p53, } \\
\text { CASP3, and COX-2 }\end{array}$ & $500 \mathrm{mg} / \mathrm{kg}$ & [103] \\
\hline & $\begin{array}{l}4 \% \text { dextran sulfate } \\
\text { sodium-induced colitis } \\
\text { in BALB/c mice }\end{array}$ & Physalin B & $\begin{array}{l}\text { Reduced MPO activity; suppressed the } \\
\text { activation of NF- } \mathrm{B} \text {, STAT3, arrestin beta } 1 \\
\text { (ARRB1), and NLR family pyrin domain } \\
\text { containing } 3 \text { (NLRP3) }\end{array}$ & $10,20 \mathrm{mg} / \mathrm{kg}$ & {$[50]$} \\
\hline & N2a/APPsw cells & Physalin B & $\begin{array}{l}\text { Downregulated } \beta \text {-amyloid }(\mathrm{A} \beta) \text { secretion } \\
\text { and the expression of beta-secretase } 1 \\
\text { (BACE1) }\end{array}$ & $3 \mu \mathrm{mol} / \mathrm{L}$ & {$[104]$} \\
\hline & $\begin{array}{l}\text { DPPH } \\
\text { TBA }\end{array}$ & Physalin D & Exhibited antioxidant activity & $\mathrm{IC}_{50} \geq 10 \pm 2.1 \mu \mathrm{g} / \mathrm{mL}$ & [92] \\
\hline & $\begin{array}{l}\text { Plasmodium } \\
\text { berghei-infected mice }\end{array}$ & Physalins B, D, F, G & Caused parasitemia reduction and delay & $50,100 \mathrm{mg} / \mathrm{kg}$ & [105] \\
\hline & $\begin{array}{l}\text { High glucose-induced } \\
\text { primary mouse } \\
\text { hepatocytes } \\
\text { Oleic acid-induced } \\
\text { HepG2 cells } \\
\text { Kunming mice }\end{array}$ & $\begin{array}{l}75 \% \text { ethanol extract } \\
\text { Luteolin-7-O- } \beta \text {-D- } \\
\text { glucopyranoside }\end{array}$ & $\begin{array}{l}\text { In vitro: decreased the levels of TG in } \\
\text { HepG2 cells } \\
\text { In vivo: decreased the levels of TC and TG }\end{array}$ & $\begin{array}{l}50,100 \mu \mathrm{g} / \mathrm{mL}, \\
\text { respectively } \\
1 \text { or } 2 \mathrm{~g} / \mathrm{kg} \text {, } \\
0.54 \mathrm{~g} / \mathrm{kg} \text {, respectively }\end{array}$ & [106] \\
\hline & SD mice & Luteolin & $\begin{array}{l}\text { Increased NO; activated PI3K/Akt/NO } \\
\text { signaling pathway; enhanced the activity } \\
\text { of endothelial NOS }\end{array}$ & $7.5 \mu \mathrm{g} / \mathrm{mL}$ & [107] \\
\hline & SD rats & Luteolin & $\begin{array}{l}\text { Conferred a cardioprotective effect; } \\
\text { ameliorated } \mathrm{Ca}^{2+} \text { overload }\end{array}$ & $7.5,15,30 \mu \mathrm{mol} / \mathrm{L}$ & [108] \\
\hline
\end{tabular}

\subsection{Anti-Inflammatory Activity}

Studies involving in vitro and in vivo models of lipopolysaccharide-stimulated (LPSstimulated) THP-1 cells, mouse ear-swelling, rat cotton pellet granuloma, and rat hind paw edema have confirmed that ethanol and methanol extracts from P. alkekengi calyxes exert anti-inflammatory effects. The extracts achieve these effects by inhibiting the production of nitric oxide (NO), prostaglandin E2 (PGE2), tumor necrosis factor- $\alpha$ (TNF- $\alpha$ ), interleukin-1 (IL-1), inducible nitric oxide synthase (iNOS), and cyclooxygenase-2 (COX-2) [62,91]. As active ingredients isolated from P. alkekengi, physalins A, B, D, E, F, H, G, L, O, V, D1, X, VII, and I, isophysalin A, isophysalin B, and aromaphysalin B showed anti-inflammatory activity. At a concentration of $20 \mu \mathrm{M}$, physalins $\mathrm{A}, \mathrm{O}, \mathrm{L}$, and $\mathrm{G}$ and isophysalin A inhibited the LPS-induced NO production by blocking TNF- $\alpha[47,48]$. Physalins B, E, F, G, H, V, X, D1, VII, and I, isophysalin B, and aromaphysalin B reduced the levels of proinflammatory mediators NO, TNF- $\alpha$, IL-6, IL-12, and interferon- $\gamma$ (IFN- $\gamma$ ) in LPS-stimulated and IFN- $\gamma$ stimulated macrophages, RAW 264.7 cells, and 12-O-tetradecanoylphorbol-13-acetate (TPA)and oxazolo-induced dermatitis. These effects occurred through upregulation of the signal transducer and activator of transcription 6 (STAT6) and downregulation of nuclear factor$\kappa \mathrm{B}(\mathrm{NF}-\mathrm{\kappa B})$ and the STAT1 signaling pathway $[48,49,51-53,55,56]$. The anti-inflammatory effects of four flavonoids (i.e., luteolin, apigenin, kaempferol, and quercetin) were related to inhibition of the production of NO, IL-6, IL-12, TNF- $\alpha$, STAT-1, and NF- $\mathrm{kB}$, the expression of $\mathrm{C}-\mathrm{C}$ motif chemokine ligand 2/monocyte chemoattractant protein-1 (CCL2/MCP-1) and $\mathrm{C}-\mathrm{X}-\mathrm{C}$ motif chemokine ligand $1 / \mathrm{KC}(\mathrm{CXCL1} / \mathrm{KC})$, and paw edema [57-60]. Ombuine inhibited the production of NO in LPS-damaged macrophage cells, with a half maximal inhibitory concentration $\left(\mathrm{IC}_{50}\right)$ value of $2.23 \pm 0.19 \mu \mathrm{M}$ [57].

\subsection{Anti-Tumor Activity}

Recently, in vitro experimental studies showed the anti-tumor activity of physalins in non-small cell lung cancer, human melanoma A375-S2 cells, and tumor cell lines (A549, $\mathrm{K} 562)$. The results indicated that physalins $\mathrm{A}$ and $\mathrm{B}$ have strong anti-tumor activity and induced G2/M cell cycle arrest in non-small cell lung cancer and A375-S2 cells. The mechanism involved in this effect is related to the inhibition of Janus kinase 2 (JAK2) phos- 
phorylation, JAK3 phosphorylation, both constitutive and induced STAT3, reactive nitrogen species (RNS), reactive oxygen species (ROS), and cyclin-dependent kinase 1/cyclin B1 (CDK1/CCNB1) complex, as well as the promotion of the p53-NADPH oxidase activator(p53-NOXA), p38-NF- $\mathrm{B}$, and p38 mitogen-activated protein kinase/ROS (MAPK/ROS) pathways $[66,67,69,70,72,73]$. Physalin A also increased the content of detoxifying enzyme in HepG2 cells, induced apoptosis in HT1080 cells, and inhibited growth in prostate cancer cells (CWR22Rv1 and C42B). These effects occurred by activating the nuclear factor erythroid 2-related factor 2-antioxidant response element (Nrf2-ARE), death receptor apoptotic, JUN N-terminal kinase (JNK), and extracellular signal-regulated kinase (ERK) signaling pathway; the $\mathrm{IC}_{50}$ values were $20,10.7,14.2$, and $1.9-4.3 \mu \mathrm{M}$, respectively $[65,67,68,71]$. In addition, six types of cancer cells (i.e., prostate, human HCT116 colon, human DLD-1 colon, breast, TNF- $\alpha$-stimulated HeLa, and human T cell leukemia Jurkat) were treated with physalin B. The treatment inhibited the activation of TNF- $\alpha$-induced NF- $\kappa$ B and phorbol 12-myristate 13-acetate (PMA)-induced NF- $\mathrm{B}$ pathways, whereas it promoted the activation of ERK, JNK, p38 MAPK, and P53 pathways [9,74-76,79]. Physalin F decreased the TOPFlash reporter activity, inhibited the effects on T-47D cells, and induced cell apoptosis via ROS-mediated mitochondrial pathways [80-82].

In vivo, physalins $A$ and $F$ clearly inhibited tumor growth by downregulating $\beta$ catenin in xenograft tumor-bearing mice [66,80]. At $10 \mathrm{mg} / \mathrm{kg}$ and $25 \mathrm{mg} / \mathrm{kg}$, respectively, physalins B and D inhibited tumor proliferation in mice bearing sarcoma 180 tumor cells [78]. In short, the anti-tumor activity of $P$. alkekengi and its constituents was associated with the downregulation of JAK/STAT3, TNF- $\alpha$-induced NF- $\kappa$ B, PMA-induced NF- $\mathrm{B}$, and phosphoinositide-3-kinase-Akt-mechanistic target of the rapamycin (PI3K/Akt/mTOR) signaling pathway. Moreover, it was linked to the upregulation of the death receptor apoptotic, p53-NOXA, p38-NF-kB, p38 MAPK/ROS, p21, and Nrf2 signaling pathway. The signaling pathways are given in Figure 5.

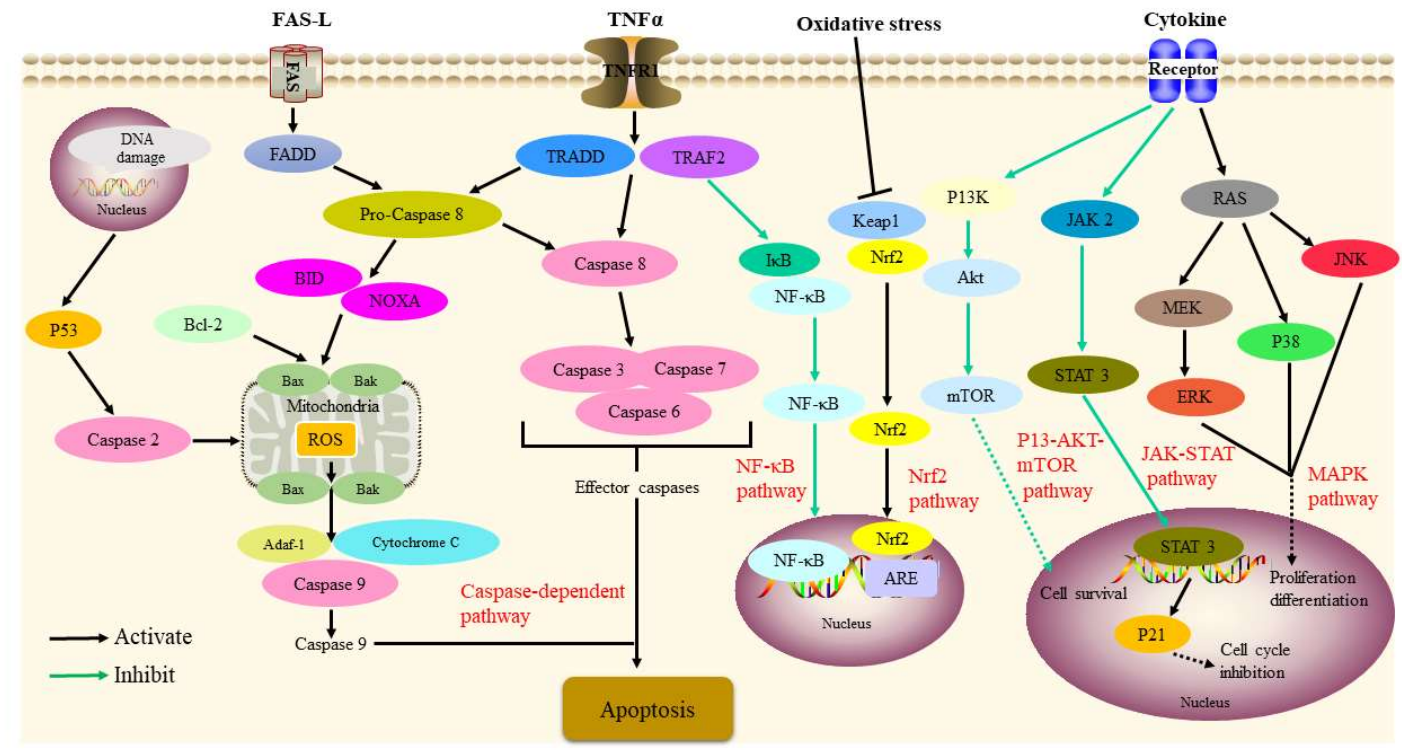

Figure 5. Signaling pathways involved in the antitumor activity of P. alkekengi and its constituents.

\subsection{Immunosuppressive Activity}

The immunosuppressive activity of P. alkekengi mainly focused on immune cells and Trypanosoma infection. Previous studies utilizing concanavalin A (Con A)-activated spleen cells suggested that physalin B inhibited Con A-induced lymphoproliferation, mixed lymphocyte reaction (MLR), and IL-2 production [88]. Yu et al. [90] found that physalin $\mathrm{H}$ also significantly inhibited the proliferation of Con A-induced T cells and MLR in vitro, with $\mathrm{IC}_{50}$ values of 0.69 and $0.39 \mu \mathrm{g} / \mathrm{mL}$, respectively. In vivo, physalin H dose-dependently in- 
hibited CD4+ T cell-mediated delayed-type hypersensitivity reactions and antigen-specific $\mathrm{T}$-cell response in ovalbumin-immunized mice, with $\mathrm{IC}_{50}$ values of $3.61 \mu \mathrm{g} / \mathrm{mL}$ for $48 \mathrm{~h}$ and $2.75 \mu \mathrm{g} / \mathrm{mL}$ for $96 \mathrm{~h}$. The mechanisms may be related to the modulation of T-helper 1/T-helper 2 (Th1/Th2) cytokine balance, inhibition of T cell activation, and proliferation and induction of HO- 1 in T cells. Moreover, at the concentration of $40 \mu \mathrm{g}$, polysaccharides from fruits of $P$. alkekengi showed good immunosuppressive effects in mice [91]. Physalin B decreased the number of T. cruzi Dm28c and T. cruzi transmission in the gut at doses of $1 \mathrm{mg} / \mathrm{mL}$ (oral administration), $20 \mathrm{ng}$ (topical application), and $57 \mathrm{ng} / \mathrm{cm}^{2}$ (contact treatment), and suppressed epimastigote forms of T. cruzi, with an $\mathrm{IC}_{50}$ value of $5.3 \pm 1.9 \mu \mathrm{M}[85,87]$. At a concentration of $1 \mu \mathrm{g} / \mathrm{mL}$, physalin B significantly increased the mortality rate (78.1\%) among Rhodnius prolixus larvae infected with Trypanosoma rangeli [86]. Physalin F prevented the rejection of allogeneic heterotopic heart transplants in vivo in a concentration-dependent manner. Moreover, it inhibited the spontaneous proliferation of peripheral blood mononuclear cells in patients with human T-cell lymphotropic virus type 1-related (HTLV1-related) myelopathy at $10 \mu \mathrm{M}$, suggesting its potential for treatments of pathologies in the inhibition of immune responses [88,89].

\subsection{Antibacterial Activity}

In vitro, at the concentration of $100 \mu \mathrm{g} / \mathrm{mL}$, physalin D isolated from P. alkekengi was found to be effective against Staphylococcus epidermidis (S. epidermidis), Enterococcus faecalis (E. faecalis), Staphylococcus aureus (S. aureus), and Bacillus subtilis (B. subtilis) [92]. Yang et al. [93] reported that physalins $\mathrm{B}, \mathrm{J}$, and $\mathrm{P}$ exhibited a good antibacterial activity against Escherichia coli (E. coli) and B. subtilis. Additionally, trichlormethane, ethanol, methanol, or aqueous extracts from $P$. alkekeng $i$ were also active against some Gram-positive and Gram-negative bacteria [62,94-96]. Janua'rio et al. [94] found that the crude trichlormethane extract (fraction A1-29-12) inhibited the Mycobacterium tuberculosis H37RV strain at a minimum concentration of $32 \mu \mathrm{g} / \mathrm{mL}$. Li et al. [95] found that the $70 \%$ ethanol extract stimulated the growth of probiotic bacteria (Lactobacillus delbrueckii) and inhibited that of pathogenic bacteria ( $E$. coli) in a dose-dependent manner. Moreover, a study indicated that physakengoses also have potent antibacterial activity against $S$. aureus, B. subtilis, and Pseudomonas aeruginosa (P. aeruginosa). The minimum inhibitory concentration (MIC) values of physakengoses $\mathrm{B}$, E, F, G, and H for $S$. aureus were $9.72 \pm 2.83,9.81 \pm 1.48,5.32 \pm 1.47,6.57 \pm 0.86$, and $5.78 \pm 0.96 \mu \mathrm{g} / \mathrm{mL}$, respectively. For $B$. subtilis, these values were $8.89 \pm 1.63,5.59 \pm 0.85$, $3.50 \pm 1.49,8.78 \pm 1.67$, and $3.57 \pm 1.02 \mu \mathrm{g} / \mathrm{mL}$, respectively. For P. aeruginosa, these values were $14.91 \pm 2.56,13.12 \pm 2.42,5.79 \pm 1.15,4.51 \pm 3.02$, and $3.21 \pm 0.95 \mu \mathrm{g} / \mathrm{mL}$, respectively [96]. Zhang et al. showed that physakengoses $\mathrm{K}, \mathrm{L}, \mathrm{M}, \mathrm{N}$, and $\mathrm{O}$ had potent antibacterial activity, with MIC values ranging from 2.16 to $12.76 \mathrm{mg} / \mathrm{mL}$ [97]. However, the mechanism involved in the antibacterial activity of P. alkekengi has not been reported yet, warranting further research. The antibacterial activity is illustrated in Figure 6.

\subsection{Antileishmanial Activity}

Physalins exhibit potent antileishmanial activity against the cutaneous leishmaniasis [109,110]. Guimarães et al. [98] reported that physalins B and F exerted in vivo antileishmanial effects in BALB/c mice infected with Leishmania amazonensis (L. amazonensis); in vitro, they demonstrated an effect against intracellular amastigotes of Leishmania. In vitro, physalins $\mathrm{B}$ and $\mathrm{F}$ inhibited the infection of macrophages with L. amazonensis, with $\mathrm{IC}_{50}$ values of 0.21 and $0.18 \mu \mathrm{M}$, respectively. Physalin F markedly reduced the lesion size and number of parasites in vivo. However, physalin D did not show this activity. This effect was associated with the inhibition of NO and proinflammatory cytokines (e.g., IL-12 and TNF- $\alpha$ ) by physalins B and F; however, physalin D lacked immunomodulatory/anti-inflammatory activity $[48,88]$. Meanwhile, the results suggest that anti-inflammatory and antileishmanial activities by physalins play a role in the treatment of cutaneous leishmaniasis. 


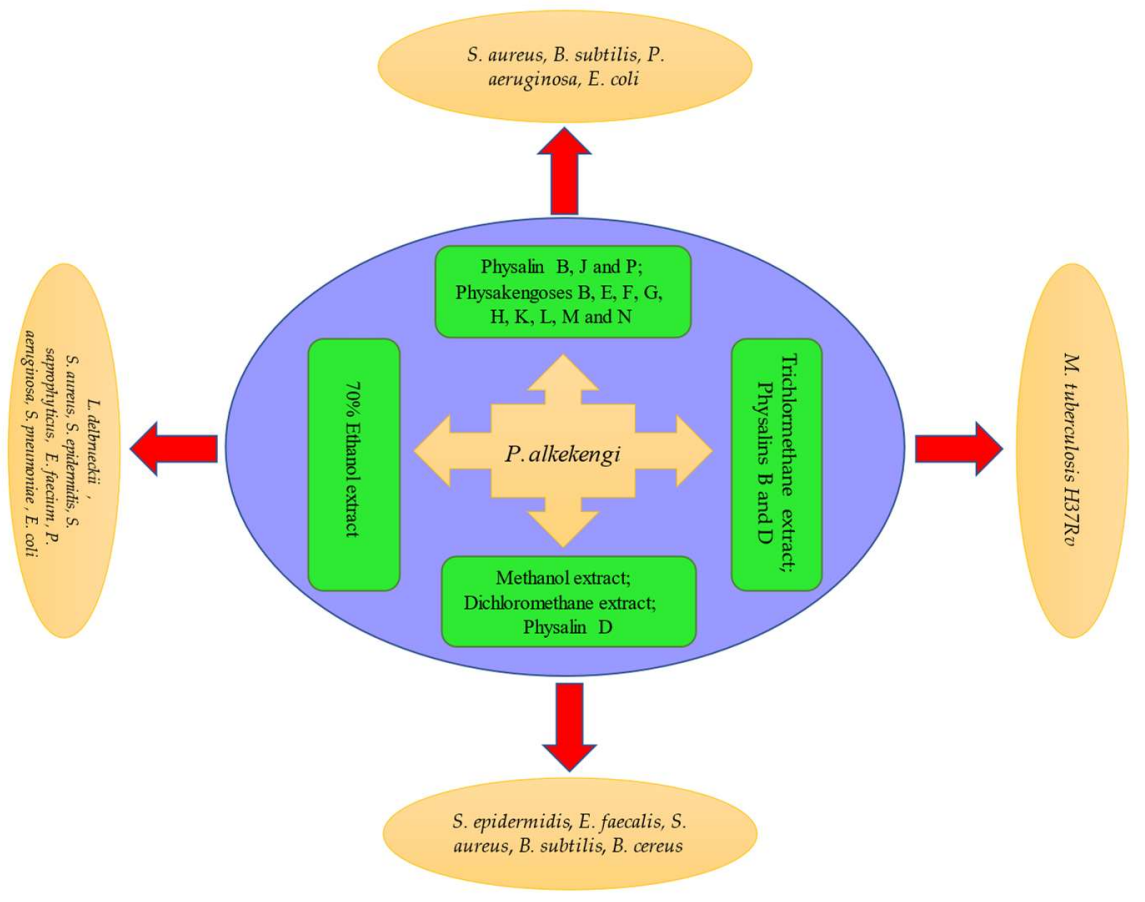

Figure 6. Schematic representation of antibacterial activity of P. alkekengi and its constituents.

\subsection{Antileishmanial Activity}

Physalins exhibit potent antileishmanial activity against the cutaneous leishmaniasis $[109,110]$. Guimarães et al. [98] reported that physalins B and F exerted in vivo antileishmanial effects in BALB/c mice infected with Leishmania amazonensis (L. amazonensis); in vitro, they demonstrated an effect against intracellular amastigotes of Leishmania. In vitro, physalins $\mathrm{B}$ and $\mathrm{F}$ inhibited the infection of macrophages with L. amazonensis, with $\mathrm{IC}_{50}$ values of 0.21 and $0.18 \mu \mathrm{M}$, respectively. Physalin F markedly reduced the lesion size and number of parasites in vivo. However, physalin D did not show this activity. This effect was associated with the inhibition of NO and proinflammatory cytokines (e.g., IL-12 and TNF- $\alpha$ ) by physalins B and F; however, physalin D lacked immunomodulatory/anti-inflammatory activity [48,88]. Meanwhile, the results suggest that anti-inflammatory and antileishmanial activities by physalins play a role in the treatment of cutaneous leishmaniasis.

\subsection{Others}

The anti-asthmatic activity of physalins has been increasingly reported over the years. In an in vitro study, following the oral administration of a water extract from P. alkekengi, the number of white blood cells and eosinophils in mice, as well as the expression of IL-5 and IFN- $\gamma$ in lung tissue, were reduced. These findings indicated its potency as a drug for the treatment of allergic asthma in children [99]. Moreover, some studies showed that luteolin effectively inhibited inflammation in asthmatic models [111]. The relevant mechanisms may be related to the inhibition of iNOS/NO signaling. Thus, more studies are required to explain the mechanisms involved in the anti-asthmatic activity of the P. alkekengi extract.

Thus far, most scientific investigations on the anti-diabetic activity of $P$. alkekengi have been carried out using the fruits, aerial parts, and polysaccharides obtained from the calyxes of $P$. alkekengi. For the fruits and aerial parts, the ethyl acetate extract effectively decreased the levels of fasting blood glucose (FBG), total cholesterol (TC), triglyceride (TG), and glycated serum protein, whereas it significantly increased those of fasting insulin (FINS) [100,102]. Moreover, polysaccharides showed anti-hyperglycemic activity on alloxan-induced mice. Although research is currently at a preliminary stage, the possible mechanisms are related to the enhancement of PI3K, Akt, and glucose transporter type 4 (GLUT4) mRNA expression, as well as the inhibition of FNG and GSP expression, 
indicating that they are promising candidates for the development of new anti-diabetic agents [101].

The anti-ulcer and anti-Helicobacter pylori effects are newly discovered pharmacological effects of $P$. alkekengi. Wang et al. reported that the P. alkekengi extract showed anti-Helicobacter pylori and gastroprotective activities by reducing the intensity of gastric mucosal damage and mitigating pain sensation [63]. It was recently reported that the $70 \%$ ethanol extract of $P$. alkekengi treated LPS-induced acute lung injury by: (1) reducing the release of TNF- $\alpha$ and the accumulation of oxidation products; (2) decreasing the levels of NF- $\mathrm{kB}$, phosphorylated-p38, ERK, JNK, p53, caspase 3 (CASP3), and COX-2; and (3) enhancing the translocation of Nrf2 from the cytoplasm to the nucleus [103]. It was also shown that the mechanism of $P$. alkekengi, which is involved in the improvement of oxidative stress damage and inflammatory response induced by acute lung injury, was related to the inhibition of NF- $\mathrm{KB}$ and the MAPK signaling pathway and the transduction of the apoptotic pathway, as well as the activation of the Nrf2 signaling pathway. Physalin $B$ could be used in the treatment of dextran sulfate sodium-induced colitis in BALB/c mice by suppressing multiple inflammatory signaling pathways [50]. In addition, physalin $B$ is effective against Alzheimer's disease through downregulation of $\beta$-amyloid $(A \beta)$ secretion and beta-secretase 1 (BACE1) expression by activating forkhead box O1 (FoxO1) and inhibiting STAT3 phosphorylation [104]. In the diphenyl-2-picrylhydrazyl (DPPH) and thiobarbituric acid (TBA) test, physalin D showed antioxidant activity, with an $\mathrm{IC}_{50}$ value $\geq 10 \pm 2.1 \mu \mathrm{g} / \mathrm{mL}$ [92]. Physalins B, D, F, and G showed low anti-plasmodial activity; nevertheless, physalin $\mathrm{D}$ markedly caused parasitemia and a delay in mortality in mice infected with Plasmodium berghei [105]. Furthermore, a study demonstrated that $75 \%$ ethanol extract of calyxes and fruits of P. alkekengi significantly decreased the serum's total cholesterol and TG levels in vivo. Moreover, luteolin-7-O- $\beta$-D-glucopyranoside isolated from $P$. alkekengi decreased the TG levels induced by oleic acid in HepG2 cells and by high glucose in primary mouse hepatocytes, thereby exhibiting hypolipidemic activity [106]. Luteolin effectively relaxed the blood vessels and preserved the rat heart, mainly through activation of the PI3K/Akt/NO signaling pathway and enhancement of the activity of endothelial NOS, as well as amelioration of the $\mathrm{Ca}^{2+}$ overload in rat cardiomyocytes $[107,108]$.

\section{Pharmacology}

\subsection{Physalins}

Absorption refers to the process by which the drug enters the blood circulation from the site of administration. Following the oral administration of the extract from the calyxes and fruits of P. alkekengi $(0.5 \mathrm{~g} / \mathrm{mL})$ in rats, liquid chromatography with MS/MS was used to investigate the pharmacokinetic profile of physalins A, D, and L (equivalent to 2, 16 , and $3 \mathrm{mg} / \mathrm{mL}$, respectively) in plasma. The results showed similar pharmacokinetic parameters for the three physalin compounds (maximum concentration: 1.3, 1.7, and $1.3 \mathrm{~h}$, respectively). The biological half-life was $2.5,3.4$, and $2.8 \mathrm{~h}$; the mean residence time was $3.6,4.9$, and $4.1 \mathrm{~h}$; and the area under curve was 113,103 , and $266 \mathrm{ng} \cdot \mathrm{h} / \mathrm{mL}$, respectively. These data revealed that the absorption characteristics of these three physalin compounds in rats were similar. Moreover, chemical structural changes in the three compounds exerted a minimal effect on the absorption rate but a greater effect on the elimination rate [112]. This is attributed to the high degree of similarity between the chemical structures of the three physalin compounds. Another study also showed that physalins A, D, and L exhibited great similarity in the time required to reach the peak concentration $(0.7,1.2$, and $0.7 \mathrm{~h}$, respectively) in rat plasma. However, isophysalin B was rarely absorbed in rats due to the conversion of gastrointestinal bacteria and metabolic enzymes through a strong first-pass effect after oral administration and its low solubility in gastrointestinal fluid [113-115]. Pharmacokinetic studies of physalins incubated with intestinal bacterial culture showed that the concentration was significantly decreased. Furthermore, most physalins could not be detected when the reaction time was increased. These results indicated that physalins are extremely unstable in rat intestinal bacteria and have low bioavailability [115]. 
Distribution refers to the process by which the drug is absorbed into the blood circulation and transported to the various organs and tissues of the body. Zheng et al. [116] revealed that, after a single intragastric administration, physalin B exhibited a single-chamber model with peak concentration of $0.08 \mathrm{~h}$ and body clearance rate by bioavailability of $0.18 \mathrm{~L} / \mathrm{min} / \mathrm{kg}$; the distribution decreased in the following order: $C_{\text {lung }}>C_{\text {heart }}>C_{\text {kidney }}>C_{\text {brain }}>C_{\text {liver }}>C_{\text {spleen }}$. The concentration of physalin $B$ in the lung was $>20$-fold higher than that measured in all other tissues, indicating that the lung is the main target organ of physalin B. Physalin B also showed significant antitumor activity against lung cancer cell lines ( $\mathrm{IC}_{50}$ value: $\left.1.2 \mu \mathrm{M}\right)$ and became a therapeutic candidate for this disease [117]. Wu et al. [118] found that physalin D was distributed and rapidly eliminated in rats within $5 \mathrm{~min}$, and the distribution characteristics in tissue decreased in the following order: $C_{\text {kidney }}>C_{\text {liver }}>C_{\text {lung }}>C_{\text {spleen }}>C_{\text {heart }}$. The highest levels were recorded in the kidney, followed by the liver; however, physalin $\mathrm{D}$ was not detected in the brain. Therefore, kidney is the major distribution tissue for physalin $\mathrm{D}$ in rats, and physalin $\mathrm{D}$ cannot cross the blood-brain barrier. This is probably because the polarity of physalin B is lower than that of physalin D, making it easier for physalin B to cross the cell membrane than physalin D.

Metabolism is also known as biotransformation; it refers to the change in the chemical structure of the drug in the body. The main metabolic reactions of physalins in the body are phase II metabolic reactions (e.g., sulfonation, acetylation, glucuronidation, etc.). Following the oral administration of calyxes and fruits of $P$. alkekengi, an analytical method based on UHPLC-Q-TOF-MS/MS was applied to identify absorbed constituents and in vivo metabolites in biological fluids obtained from rats. The results identified 33 compounds in vivo: 12 and 21 compounds were predicted to be prototype components and metabolites of $P$. alkekengi, respectively. Lastly, sulfonation and hydroxylation were recognized as the metabolic pathways for physalin constituents [119]. Another study focused on the metabolism of physalin A in rats after oral administration. A total of 24 proposed metabolites were identified in the plasma, bile, urine, and feces. The major metabolic pathways of physalin A in the body were sulfonation, reduction, and hydroxylation. These analyses provided a framework for studying the possible metabolic pathways of other physalins and evaluating the relationship of metabolites with parent compounds in the context of the internal environment [120].

Excretion refers to the process through which the prototype of a drug or its metabolites are transported out of the body through excretory or secretory organs. A rapid and sensitive method was developed to investigate urine and feces samples collected at different exposure times after the oral administration of physalin D $(25 \mathrm{mg} / \mathrm{kg})$. The analysis showed that $12.26 \%$ of the orally administered dose of physalin D was excreted in the feces, in an unchanged form, within $72 \mathrm{~h}$. The physalin D in feces was mainly excreted within 12-24 h, and the excretion ratio in feces decreased in parallel with the decreasing concentration of physalin D in the rat. Physalin D in urine was mainly excreted in the form of glucuronide and sulfate, mainly within 4-36 h, and the amount decreased with time. The excretion data of physalin D in urine and feces indicated that $<14.0 \%$ of the administered dose was excreted in an unconverted form. These results revealed that physalin D was extensively and rapidly metabolized in rats after intragastric administration, leading to a short biological halflife [121]. The pharmacokinetics of physalins are shown in Table 3. 
Table 3. Summary of the pharmacokinetic parameters of physalins in rat plasma after single oral administration of $P$. alkekengi.

\begin{tabular}{|c|c|c|c|c|c|c|c|c|c|c|c|}
\hline Methods & Compounds & $\begin{array}{l}\text { Dose/ } \\
\mathrm{mg} / \mathrm{kg}\end{array}$ & $t_{1 / 2} / h$ & $\begin{array}{l}\mathrm{C}_{\max } / \\
\mathrm{ng} / \mathrm{mL}\end{array}$ & $\mathrm{T}_{\max } / \mathrm{h}$ & $\begin{array}{l}\mathrm{CL} / \mathrm{L} / \\
\mathrm{min} / \mathrm{kg}\end{array}$ & $\mathrm{MRT}_{0-\mathrm{t}} / \mathrm{h}$ & $\mathrm{MRT}_{0-\infty} / \mathrm{h}$ & $\begin{array}{l}\mathrm{AUC}_{0-\mathrm{t}} / \\
\mathrm{ng} \cdot \mathrm{h} / \mathrm{mL}\end{array}$ & $\begin{array}{l}\mathrm{AUC}_{0-\infty} / \\
\mathrm{ng} \cdot \mathrm{h} / \mathrm{mL}\end{array}$ & Reference \\
\hline LC-MS/MS & $\begin{array}{l}\text { Physalin A } \\
\text { Physalin D } \\
\text { Physalin L }\end{array}$ & $\begin{array}{l}2 \\
16 \\
3\end{array}$ & $\begin{array}{l}2.52 \pm 0.40 \\
3.36 \pm 0.26 \\
2.82 \pm 0.25\end{array}$ & $\begin{array}{l}5.30 \pm 1.76 \\
11.5 \pm 3.57 \\
56.4 \pm 15.4\end{array}$ & $\begin{array}{l}1.29 \pm 2.31 \\
1.67 \pm 1.46 \\
1.28 \pm 1.33\end{array}$ & $\begin{array}{l}- \\
-\end{array}$ & $\begin{array}{l}3.63 \pm 0.57 \\
4.85 \pm 0.37 \\
4.07 \pm 0.37\end{array}$ & - & $\begin{array}{l}21.0 \pm 3.14 \\
70.5 \pm 10.10 \\
200 \pm 31.30\end{array}$ & $\begin{array}{l}113 \pm 103 \\
103 \pm 30.2 \\
266 \pm 53.0\end{array}$ & [112] \\
\hline $\begin{array}{l}\text { UPLC- } \\
\text { MS/MS }\end{array}$ & $\begin{array}{l}\text { Physalin D } \\
\text { Physalin G } \\
\text { 4,7-Didehydro- } \\
\text { neophysalin B }\end{array}$ & $\begin{array}{l}35.6 \\
13.9 \\
32.6\end{array}$ & $\begin{array}{l}3.67 \pm 1.04 \\
8.04 \pm 3.42 \\
6.15 \pm 1.20\end{array}$ & $\begin{array}{l}47.6 \pm 4.10 \\
20.9 \pm 4.40 \\
23.6 \pm 4.90\end{array}$ & $\begin{array}{l}1.17 \pm 0.00 \\
1.17 \pm 0.00 \\
1.17 \pm 0.00\end{array}$ & $\begin{array}{l}4.4 \pm 0.60 \\
3.2 \pm 0.70 \\
8.7 \pm 1.90\end{array}$ & $\begin{array}{l}3.42 \pm 0.33 \\
4.69 \pm 1.41 \\
4.89 \pm 0.43\end{array}$ & - & $\begin{array}{l}60.82 \pm 14.32 \\
61.24 \pm 11.53 \\
60.82 \pm 12.85\end{array}$ & $\begin{array}{l}136.94 \pm 17.18 \\
74.56 \pm 17.46 \\
64.82 \pm 14.80\end{array}$ & [113] \\
\hline LC-MS/MS & Physalin L & 18.52 & $2.89 \pm 1.14$ & $77.48 \pm 28.30$ & $0.69 \pm 0.26$ & $50.26 \pm 11.50$ & $3.13 \pm 0.63$ & $4.33 \pm 1.50$ & $280.78 \pm 86.48$ & $313.10 \pm 101.24$ & [114] \\
\hline $\begin{array}{l}\text { HPLC- } \\
\text { MS/MS }\end{array}$ & Physalin B & 5 & $5.35 \pm 0.49$ & $395.0 \pm 35.4$ & $0.08 \pm 0.0$ & $0.18 \pm 0.03$ & - & - & $382.25 \pm 24.87$ & $449.92 \pm 27.46$ & [116] \\
\hline $\begin{array}{l}\text { HPLC- } \\
\text { MS/MS }\end{array}$ & Physalin D & 2 & $0.09 \pm 0.07$ & $941.3 \pm 272.1$ & $0.08 \pm 0.0$ & $0.12 \pm 0.01$ & $0.30 \pm 0.12$ & - & $28.30 \pm 29.02$ & $283.89 \pm 28.37$ & [118] \\
\hline $\begin{array}{l}\text { SPE-LC- } \\
\text { MS/MS }\end{array}$ & $\begin{array}{l}\text { Physalin A } \\
\text { Physalin D } \\
\text { Physalin G } \\
\text { 4,7-Didehydro- } \\
\text { neophysalin B }\end{array}$ & $\begin{array}{l}29 \\
38.8 \\
18.3 \\
31.6\end{array}$ & $\begin{array}{l}1.83 \pm 0.61 \\
3.11 \pm 1.37 \\
2.24 \pm 1.47 \\
2.32 \pm 1.01\end{array}$ & $\begin{array}{l}12.73 \pm 2.08 \\
64.58 \pm 21.30 \\
89.93 \pm 26.05 \\
19.63 \pm 7.21\end{array}$ & $\begin{array}{l}0.67 \pm 0.15 \\
1.29 \pm 0.78 \\
0.67 \pm 0.00 \\
1.13 \pm 0.32\end{array}$ & - & - & - & $\begin{array}{l}65.21 \pm 10.52 \\
615.39 \pm 97.86 \\
159.12 \pm 34.76 \\
105.5 \pm 28.21\end{array}$ & $\begin{array}{l}96.31 \pm 30.50 \\
885.18 \pm 230.68 \\
205.07 \pm 49.8 \\
173.58 \pm 17.90\end{array}$ & [115] \\
\hline
\end{tabular}

Abbreviation: AUC, area under curve; $C L$, clearance rate; $C_{\max }$, maximum concentration; $M R T$, mean residence time; $\mathrm{t}_{1 / 2}$, biological half-life; $\mathrm{T}_{\max }$, peak concentration.

\subsection{Flavonoids}

Flavonoids are widely distributed in the calyxes and fruits of $P$. alkekengi and exhibit anti-allergic, anti-inflammatory, antioxidant, and inhibitory effects on $\mathrm{NO}$ as the main active ingredient [122]. However, the in vivo absorption of flavonoids has been rarely investigated. Guo et al. [112] conducted a pharmacokinetic characterization of luteolin7-O-glucopyranoside and luteolin. They found that the plasma concentrations of two flavonoids could not reach the lower limit of quantitation at most timepoints. Small amounts of compounds were detected due to the relatively low levels of flavonoids ( 1 and $0.4 \mathrm{mg} / \mathrm{g}$, respectively) in extracts from the calyxes and fruits of P. alkekengi. Moreover, evidence suggested that flavonoids were usually consumed in the small intestine as a proportion of aglycone [123]. Luteolin is produced by the metabolism of luteoloside. Subsequently, it may be transported to the liver through the portal vein, where it may form a potential phase I substrate through further hydroxylation in the liver. Consequently, it produces more polar compounds through further phase I and II metabolism [124,125], such as the glucuronidation of luteolin and the hydroxylation and sulfation of other types of flavonoids [119]. Therefore, the content of prototype constituents in the body would be significantly decreased or undetectable.

\section{Conclusions and Future Perspectives}

Thus far, $>170$ compounds have been isolated and identified from P. alkekengi; the most common are physalins, flavonoids, sucrose esters, and other trace elements [5]. Among these ingredients, 18 new compounds were isolated from the P. alkekengi, including nine steroids ( $7 \alpha$-hydroxy-5-deoxy-4-dehydrophysalin IX, 5-deoxy-4-dehydrophysalin IX, $7 \beta$-ethoxyl-isophysalin C, etc.) $[77,97,126,127]$. Numerous pharmacological studies have revealed various biological properties of $P$. alkekengi (i.e., anti-inflammatory, anticancer, immunosuppressive, anti-leishmanial, anti-asthmatic, anti-diabetic, antioxidative, anti-malarial, anti-vasodilatory, anti-colitic, anti-ulcer, acting as febricide, expectorant, or diuretic, etc.). Physalins and flavonoids are closely related to the pharmacological activity of P. alkekengi. Accordingly, further study is urgently needed to gain a better understanding of $P$. alkekengi and its clinical use.

Firstly, this review summarizes the structural analysis of natural products of physalins and flavonoids. Physalins are synthesized in P. alkekengi via MEV and MEP pathways, and flavonoids are synthesized via phenylpropanoid pathway. However, apart from some studies on the physalins' skeleton of natural products, there is almost no research conducted on the synthesis of specific physalins and their derivatives. The importance of the right-side (DFGH-ring) structure of physalins for biological activity is established. For 
example, synthesis of DFGH-ring derivatives of physalins with a hydrophobic substituent is important for the inhibitory activity [128]. Therefore, there is a need for further exploration of the synthesis of physalins and development of more clinically valuable compounds.

Secondly, a holistic quality control method that is correlated with the pharmacological effects of $P$. alkekengi is warranted. Current quality control methods are mainly focused on HPLC, and it is difficult to distinguish genuine products from counterfeit goods. The purpose of quality control in TCM is to monitor effective substances and their variations in the production process. By summarizing the currently available literature, we found that the contents of bioactive compounds differ significantly in samples obtained from different sources and at different collection times. Therefore, safe, high-quality, and high efficiency planting techniques for this plant should be further investigated to guide its production for TCM.

Thirdly, previous pharmacological investigations on $P$. alkekengi have yielded considerable evidence regarding its anti-inflammatory and anti-cancer properties and have elucidated the mechanisms of their action in vitro and in vivo. However, few studies concentrated on its immunosuppressive, anti-leishmanial, anti-asthmatic, anti-diabetic, antioxidative, anti-malarial, anti-vasodilatory, and anti-colitic effects, which warrant further exploration. Additionally, several studies have highlighted the potential of P. alkekengi as a novel therapeutic agent for the treatment of ulcers, Helicobacter pylori, LPS-induced induced acute lung injury, and Alzheimer's disease. Nevertheless, the mechanism underlying these treatment effects should be fully elucidated using current techniques.

Lastly, the absorption, distribution, metabolism, and excretion of physalins in the body are explained. These compounds are characterized by fast absorption, wide distribution, and rapid excretion. These findings indicated that physalins are extremely unstable and have low bioavailability in the intestine. Hence, they must overcome certain factors that control the sustained and stable release of drugs in the blood and improve oral bioavailability, thereby exerting good pharmacological effects. Unfortunately, however, it should be noted that few studies have investigated the pharmacokinetics of extracts and active compounds, particularly flavonoids. Consequently, further clinical application of $P$. alkekengi may be limited until further pharmacokinetics studies in the laboratory and clinic are performed.

In summary, P. alkekeng $i$ is an excellent, abundant, inexpensive, and edible drug. The synthesis of the main active components of $P$. alkekengi must be further analyzed using additional biological and chemical techniques to further expand their potential applications. In addition, the quantitative analysis of the chemical constituents of P. alkekengi should be employed for the purpose of standardization and quality control of extracts. Lastly, additional in vivo animal research and clinical trials are needed to determine whether various applications of $P$. alkekengi are effective and safe in a larger population.

Author Contributions: Conceptualization, H.K. and B.Y.; writing-original draft preparation, J.Y.; writing - review and editing, F.C. and Y.S. All authors have read and agreed to the published version of the manuscript.

Funding: This research was supported by the Heilongjiang Touyan Innovation Team Program, QiHuang Scholar of National Traditional Chinese Medicine Leading Talents Support Program (2018), and Chief Scientist of Qi-Huang Project of National Traditional Chinese Medicine Inheritance and Innovation “One Hundred Million” Talent Project (2021).

Institutional Review Board Statement: Not applicable.

Informed Consent Statement: Not applicable.

Data Availability Statement: All reported or analyzed data in this review are extracted from published articles.

Conflicts of Interest: The authors declare no conflict of interest. 


\section{References}

1. Chinese Pharmacopoeia Commission. Pharmacopoeia of the People's Republic of China Part I; People's Medical Publishing House: Beijing, China, 2020; p. 360. (In Chinese)

2. Zheng, W.J.; Fu, L.G. Flora of China; Editorial Committee of Flora of China, Chinese Academy of Sciences, Science Press: Beijing, China, 1978; p. 54. (In Chinese)

3. Shu, Z.P.; Xu, B.Q.; Xing, N.; Li, X.L.; Wang, Q.H.; Yang, B.Y.; Kuang, H.X. Chemical constituents of Physalis Calyx seu Fructus. Zhong Guo Shi Yan Fang Ji Xue Za Zhi 2014, 20, 99-102.

4. Gao, P.Y.; Jin, M.; Du, C.L.; Liu, X.G. Research progress of Physalis alkekengi var. franchetii. Shenyang Yao Ke Da Xue Xue Bao 2014, 31, 732-737.

5. Wen, X.; Erşan, S.; Li, M.; Wang, K.; Steingass, C.B.; Schweiggert, R.M.; Ni, Y.; Carle, R. Physicochemical characteristics and phytochemical profiles of yellow and red Physalis (Physalis alkekengi L. and P.pubescens L.) fruits cultivated in China. Food Res. Int. 2019, 120, 389-398. [CrossRef]

6. $\quad$ Li, A.L.; Chen, B.J.; Li, G.H.; Zhou, M.X.; Li, Y.R.; Ren, D.M.; Lou, H.X.; Wang, X.N.; Shen, T. Physalis alkekengi L. var. franchetii (Mast.) Makino: An ethnomedical, phytochemical and pharmacological review. J. Ethnopharmacol. 2018, 210, 260-274. [CrossRef]

7. Yang, L.J.; Wang, D.D.; Wu, H.J.; Chen, D.Z. Study on the action targets for anti-inflammatory bioactive components of Physalis alkekengi L. var. franchetii (Mast.) Makino based on network pharmacology. J. Tianjin Univ. Tradit. Chin. Med. 2018, 37, $399-403$.

8. Huang, M.; He, J.X.; Hu, H.X.; Zhang, K.; Wang, X.N.; Zhao, B.B.; Lou, H.X.; Ren, D.M.; Shen, T. Withanolides from the genus Physalis: A review on their phytochemical and pharmacological aspects. J. Pharm. Pharmacol. 2020, 72, 649-669. [CrossRef]

9. Ozawa, M.; Morita, M.; Hirai, G.; Tamura, S.; Kawai, M.; Tsuchiya, A.; Oonuma, K.; Maruoka, K.; Sodeoka, M. Contribution of Cage-Shaped Structure of Physalins to Their Mode of Action in Inhibition of NF-kB Activation. ACS Med. Chem. Lett. 2013, 4, 730-735. [CrossRef] [PubMed]

10. Wu, J.; Zhao, J.; Zhang, T.; Gu, Y.; Khan, I.A.; Zou, Z.; Xu, Q. Naturally occurring physalins from the genus Physalis: A review. Phytochemistry 2021, 191, 112925. [CrossRef]

11. Pandey, S.S.; Singh, S.; Pandey, H.; Srivastava, M.; Ray, T.; Soni, S.; Pandey, A.; Shanker, K.; Babu, C.S.V.; Banerjee, S.; et al. Endophytes of Withania somnifera modulate in planta content and the site of withanolide biosynthesis. Sci. Rep. 2018, 8, 5450. [CrossRef]

12. Dubey, V.S.; Bhalla, R.; Luthra, R. An overview of the non-mevalonate pathway for terpenoid biosynthesis in plants. J. Biosci. 2003, 28, 637-646. [CrossRef]

13. Kushwaha, R.K.; Singh, S.; Pandey, S.S.; Kalra, A.; Babu, C.S.V. Fungal endophytes attune withanolide biosynthesis in Withania somnifera, prime to enhanced withanolide A content in leaves and roots. World J. Microbiol. Biotechnol. 2019, 35, 20. [CrossRef]

14. Singh, S.; Pal, S.; Shanker, K.; Chanotiya, C.S.; Gupta, M.M.; Dwivedi, U.N.; Shasany, A.K. Sterol partitioning by HMGR and DXR for routing intermediates toward withanolide biosynthesis. Physiol. Plant. 2014, 152, 617-633. [CrossRef] [PubMed]

15. Gupta, P.; Goel, R.; Pathak, S.; Srivastava, A.; Singh, S.P.; Sangwan, R.S.; Asif, M.H.; Trivedi, P.K. De novo assembly, functional annotation and comparative analysis of Withania somnifera leaf and root transcriptomes to identify putative genes involved in the withanolides biosynthesis. PLoS ONE 2013, 8, e62714. [CrossRef]

16. Singh, G.; Tiwari, M.; Singh, S.P.; Singh, S.; Trivedi, P.K.; Misra, P. Silencing of sterol glycosyltransferases modulates the withanolide biosynthesis and leads to compromised basal immunity of Withania somnifera. Sci. Rep. 2016, 6, 25562. [CrossRef]

17. Sharma, A.; Rather, G.A.; Misra, P.; Dhar, M.K.; Lattoo, S.K. Jasmonate responsive transcription factor WsMYC2 regulates the biosynthesis of triterpenoid withanolides and phytosterol via key pathway genes in Withania somnifera (L.) Dunal. Plant Mol. Biol. 2019, 100, 543-560. [CrossRef] [PubMed]

18. Morita, M.; Kojima, S.; Ohkubo, M.; Koshino, H.; Hashizume, D.; Hirai, G.; Maruoka, K.; Sodeoka, M. Synthesis of the right-side structure of type B physalins. Isr. J. Chem. 2017, 57, 309-318. [CrossRef] [PubMed]

19. Ohkubo, M.; Hirai, G.; Sodeoka, M. Synthesis of the DFGH ring system of type B physalins: Highly oxygenated, cage-shaped molecules. Angew. Chem. 2009, 48, 3862-3866. [CrossRef]

20. Morita, M.; Hirai, G.; Ohkubo, M.; Koshino, H.; Hashizume, D.; Maruoka, K.; Sodeoka, M. Kinetically controlled one-pot formation of DEFGH-rings of type B physalins through domino-type transformations. Org. Lett. 2012, 14, 3434-3437. [CrossRef]

21. Gupta, P.; Agarwal, A.V.; Akhtar, N.; Sangwan, R.S.; Singh, S.P.; Trivedi, P.K. Cloning and characterization of 2-C-methyl-Derythritol-4-phosphate pathway genes for isoprenoid biosynthesis from Indian ginseng, Withania somnifera. Protoplasma 2013, 250, 285-295. [CrossRef]

22. Kim, B.G.; Yang, S.M.; Kim, S.Y.; Cha, M.N.; Ahn, J.H. Biosynthesis and production of glycosylated flavonoids in Escherichia coli: Current state and perspectives. Appl. Microbiol. Biotechnol. 2015, 99, 2979-2988. [CrossRef]

23. Qiu, L.; Jiang, Z.H.; Liu, H.X.; Chen, L.X.; Qu, G.X.; Qiu, F. Flavonoid glycosides of the calyx Physalis. J. Shenyang Pharm. Univ. 2007, 24, 744-747.

24. Vogt, T. Phenylpropanoid biosynthesis. Mol. Plant 2010, 3, 2-20. [CrossRef]

25. Zou, L.Q.; Wang, C.X.; Kuang, X.J.; Li, Y.; Sun, C. Advance in flavonoids biosynthetic pathway and synthetic biology. China J. Chin. Mater. Med. 2016, 22, 4124-4128.

26. Falcone Ferreyra, M.L.; Rius, S.P.; Casati, P. Flavonoids: Biosynthesis, biological functions, and biotechnological applications. Front. Plant Sci. 2012, 3, 222. [CrossRef] 
27. Petrussa, E.; Braidot, E.; Zancani, M.; Peresson, C.; Bertolini, A.; Patui, S.; Vianello, A. Plant flavonoids—biosynthesis, transport and involvement in stress responses. Int. J. Mol. Sci. 2013, 14, 14950-14973. [CrossRef]

28. Liu, Y.Y.; Chen, X.R.; Wang, J.P.; Cui, W.Q.; Xing, X.X.; Chen, X.Y.; Ding, W.Y.; God'spower, B.O.; Eliphaz, N.; Sun, M.Q.; et al Transcriptomic analysis reveals flavonoid biosynthesis of Syringa oblata Lindl. in response to different light intensity. BMC Plant Biol. 2019, 19, 487. [CrossRef]

29. Zhai, R.; Liu, X.T.; Feng, W.T.; Chen, S.S.; Xu, L.F.; Wang, Z.G.; Zhang, J.L.; Li, P.M.; Ma, F.W. Different biosynthesis patterns among flavonoid 3-glycosides with distinct effects on accumulation of other flavonoid metabolites in pears (Pyrus bretschneideri Rehd.). PLoS ONE 2014, 9, e91945. [CrossRef]

30. Yu, X.; Zhu, Y.; Fan, J.; Wang, D.; Gong, X.; Ouyang, Z. Accumulation of flavonoid glycosides and UFGT gene expression in mulberry leaves (Morus alba L.) before and after frost. Chem. Biodivers. 2017, 14, e1600496. [CrossRef] [PubMed]

31. Jiang, L.; Zou, M.; Zhao, S.; Sun, Y.J.; Xu, B.L. Investigated the germplasm resources of Physalis alkekengi L. Res. Pract. Chin. Med. 2019, 33, 16-19.

32. Laczkó-Zöld, E.; Forgó, P.; Zupkó, I.; Sigrid, E.; Hohmann, J. Content determination of physalins in Physalis alkekengi by HPLC. J. China Pharm. 2011, 22, 1393-1395.

33. Xu, B.L.; Li, X.K.; Wang, B. Simultaneous determination of five components in Physalis Calyx seu Fructus by HPLC. Chin. Tradit. Pat. Med. 2014, 36, 1700-1705.

34. Yu, X.; Xu, B.L. ISSR analysis for genetic diversity of Physalis Calyx seu Fructus in different growing environment. Res. Pract. Chin. Med. 2017, 31, 15-17.

35. Laczkó-Zöld, E.; Forgó, P.; Zupkó, I.; Sigrid, E.; Hohmann, J. Isolation and quantitative analysis of physalin D in the fruit and calyx of Physalis alkekengi L. Acta Biol. Hung. 2017, 68, 300-309. [CrossRef]

36. Kranjc, E.; Albreht, A.; Vovk, I.; Glavnik, V. High performance thin-layer chromatography-mass spectrometry enables reliable analysis of physalins in different plant parts of Physalis alkekengi L. J. Chromatogr. A 2017, 1526, 137-150. [CrossRef] [PubMed]

37. Zheng, Y.; Luan, L.; Chen, Y.; Ren, Y.; Wu, Y. Characterization of physalins and fingerprint analysis for the quality evaluation of Physalis alkekengi L. var. franchetii by ultra-performance liquid chromatography combined with diode array detection and electrospray ionization tandem mass spectrometry. J. Pharm. Biomed. Anal. 2012, 71, 54-62. [CrossRef]

38. Huang, C.; Xu, Q.; Chen, C.; Song, C.; Xu, Y.; Xiang, Y.; Feng, Y.; Ouyang, H.; Zhang, Y.; Jiang, H. The rapid discovery and identification of physalins in the calyx of Physalis alkekengi L. var. franchetii (Mast.) Makino using ultra-high performance liquid chromatography-quadrupole time of flight tandem mass spectrometry together with a novel three-step data mining strategy. $J$. Chromatogr. A 2014, 1361, 139-152. [PubMed]

39. Zheng, Y.; Chen, Y.; Ren, Y.; Luan, L.; Wu, Y. Quantitative and transformation product analysis of major active physalins from Physalis alkekengi var. franchetii (Chinese Lantern) using ultraperformance liquid chromatography with electrospray ionisation tandem mass spectrometry and time-of-flight mass. Phytochem. Anal. 2012, 23, 337-344. [CrossRef] [PubMed]

40. Yu, T.; Liu, Y.Q.; Mu, C.X.; Feng, X.; Zhao, H.D.; Lin, C.X.; Cai, Q. Determination of 4,7-didehydro-neophysalin B in the fruits of Physalis alkekengi L. var. franchetii (mast.) Mskino by HPLC. Liaoning J. Tradit. Chin. Med. 2008, 35, 584-585.

41. Zhao, H.D.; Lin, C.X.; Yin, C.X.; Yu, T.; Feng, X.; Cai, Q. Determination of 4,7-didehydro-neophysalinB in Physalis alkekengi L. var. franchetii (mast.) Mskino calyces by HPLC. J. Liaoning Univ. Tradit. Chin. Med. 2008, 10, 129-130.

42. Cheng, X.M.; Zhang, C.H.; Chou, G.X.; Wang, Z.T. Investigation on quality standard of franchet groundcherry. China J. Chin. Mater. Medica 2010, 35, 2103-2105.

43. Xu, B.L.; Li, X.K.; Wang, B. Change for sugar compositionin Physalis Calyx seu Fructus from different areas. Liaoning J. Tradit. Chin. Med. 2014, 41, 988-991.

44. Wen, X.; Hempel, J.; Schweiggert, R.M.; Ni, Y.; Carle, R. Carotenoids and carotenoid esters of red and yellow physalis (Physalis alkekengi L. and P. pubescens L.) fruits and calyces. J. Agric. Food Chem. 2017, 65, 6140-6151. [CrossRef]

45. Wang, H.P.; Zhang, X.Y.; Song, X.B.; Li, Y.F.; Li, Q.H. Content determination of luteolin in Physalis Permviana Liquid by HPLC Heilongjiang Med. 2004, 17, 10-11.

46. Zhang, G.S.; Zhao, Y.L.; Yan, J.J.; Yu, Z.G. Simultaneous determination of 7 kinds of flavonoids in Jinhuang yanyan tablets by HPLC. J. Shenyang Pharm. Univ. 2012, 29, 693-696.

47. Ji, L.; Yuan, Y.; Luo, L.; Chen, Z.; Ma, X.; Ma, Z.; Cheng, L. Physalins with anti-inflammatory activity are present in Physalis alkekengi var. franchetii and can function as michael reaction acceptors. Steroids 2012, 77, 441-447. [CrossRef]

48. Soares, M.B.; Bellintani, M.C.; Ribeiro, I.M.; Tomassini, T.C.; dos Santos, R.R. Inhibition of macrophage activation and lipopolysaccaride-induced death by seco-steroids purified from Physalis angulata L. Eur. J. Pharmacol. 2003, 459, 107-112. [CrossRef]

49. Vieira, A.T.; Pinho, V.; Lepsch, L.B.; Scavone, C.; Ribeiro, I.M.; Tomassini, T.; Ribeiro-dos-Santos, R.; Soares, M.B.; Teixeira, M.M.; Souza, D.G. Mechanisms of the anti-inflammatory effects of the natural secosteroids physalins in a model of intestinal ischaemia and reperfusion injury. Br. J. Pharmacol. 2005, 146, 244-251. [CrossRef] [PubMed]

50. Zhang, Q.; Xu, N.; Hu, X.; Zheng, Y. Anti-colitic effects of physalin B on dextran sodium sulfate-induced BALB/c mice by suppressing multiple inflammatory signaling pathways. J. Ethnopharmacol. 2020, 259, 112956. [CrossRef] [PubMed]

51. Ding, N.; Wang, Y.; Dou, C.; Liu, F.; Guan, G.; Wei, K.; Yang, J.; Yang, M.; Tan, J.; Zeng, W.; et al. Physalin D regulates macrophage M1/M2 polarization via the STAT1/6 pathway. J. Cell. Physiol. 2019, 234, 8788-8796. [CrossRef] [PubMed] 
52. Yang, Y.J.; Yi, L.; Wang, Q.; Xie, B.B.; Dong, Y.; Sha, C.W. Anti-inflammatory effects of physalin E from Physalis angulata on lipopolysaccharide-stimulated RAW 264.7 cells through inhibition of NF-kB pathway. Immunopharmacol. Immunotoxicol. 2017, 39, 74-79. [CrossRef] [PubMed]

53. Pinto, N.B.; Morais, T.C.; Carvalho, K.M.; Silva, C.R.; Andrade, G.M.; Brito, G.A.; Veras, M.L.; Pessoa, O.D.; Rao, V.S.; Santos, F.A. Topical anti-inflammatory potential of physalin E from Physalis angulata on experimental dermatitis in mice. Phytomedicine 2010, 17, 740-743. [CrossRef]

54. Brustolim, D.; Vasconcelos, J.F.; Freitas, L.A.; Teixeira, M.M.; Farias, M.T.; Ribeiro, Y.M.; Tomassini, T.C.; Oliveira, G.G.; Pontes-deCarvalho, L.C.; Ribeiro-dos-Santos, R.; et al. Activity of physalin F in a collagen-induced arthritis model. J. Nat. Prod. 2010, 73, 1323-1326. [CrossRef]

55. Sun, C.P.; Oppong, M.B.; Zhao, F.; Chen, L.X.; Qiu, F. Unprecedented 22,26-seco physalins from Physalis angulata and their anti-inflammatory potential. Org. Biomol. Chem. 2017, 15, 8700-8704. [CrossRef]

56. Sun, C.P.; Qiu, C.Y.; Zhao, F.; Kang, N.; Chen, L.X.; Qiu, F. Physalins V-IX, 16,24-cyclo-13,14-seco withanolides from Physalis angulata and their antiproliferative and anti-inflammatory activities. Sci. Rep. 2017, 7, 4057. [CrossRef] [PubMed]

57. Qiu, L.; Zhao, F.; Jiang, Z.H.; Chen, L.X.; Zhao, Q.; Liu, H.X.; Yao, X.S.; Qiu, F. Steroids and flavonoids from Physalis alkekengi var. franchetii and their inhibitory effects on nitric oxide production. J. Nat. Prod. 2008, 71, 642-646. [CrossRef] [PubMed]

58. Ziyan, L.; Yongmei, Z.; Nan, Z.; Ning, T.; Baolin, L. Evaluation of the anti-inflammatory activity of luteolin in experimental animal models. Planta Medica 2007, 73, 221-226. [CrossRef] [PubMed]

59. Funakoshi-Tago, M.; Nakamura, K.; Tago, K.; Mashino, T.; Kasahara, T. Anti-inflammatory activity of structurally related flavonoids, apigenin, luteolin and fisetin. Int. Immunopharmacol. 2011, 11, 1150-1159. [CrossRef]

60. Hämäläinen, M.; Nieminen, R.; Vuorela, P.; Heinonen, M.; Moilanen, E. Anti-inflammatory effects of flavonoids: Genistein, kaempferol, quercetin, and daidzein inhibit STAT-1 and NF- $\mathrm{kB}$ activations, whereas flavone, isorhamnetin, naringenin, and pelargonidin inhibit only NF- $\mathrm{BB}$ activation along with their inhibitory effect on iNOS expression and NO production in activated macrophages. Mediat. Inflamm. 2007, 2007, 045673.

61. Kim, H.P.; Son, K.H.; Chang, H.W.; Kang, S.S. Anti-inflammatory plant flavonoids and cellular action mechanisms. J. Pharmacol. Sci. 2004, 96, 229-245. [CrossRef] [PubMed]

62. Shu, Z.; Xing, N.; Wang, Q.; Li, X.; Xu, B.; Li, Z.; Kuang, H.X. Antibacterial and anti-inflammatory activities of Physalis alkekengi var. franchetii and its main constituents. Evid.-Based Complement. Altern. Med. 2016, 2016, 4359394. [CrossRef] [PubMed]

63. Wang, Y.; Wang, S.L.; Zhang, J.Y.; Song, X.N.; Zhang, Z.Y.; Li, J.F.; Li, S. Anti-ulcer and anti-Helicobacter pylori potentials of the ethyl acetate fraction of Physalis alkekengi L. var. franchetii (Solanaceae) in rodent. J. Ethnopharmacol. 2018, 211, 197-206. [CrossRef]

64. Qiu, L.; Zhao, F.; Liu, H.; Chen, L.; Jiang, Z.; Liu, H.; Wang, N.; Yao, X.; Qiu, F. Two New Megastigmane Glycosides, Physanosides A and B, from Physalisalkekengi L. var. franchetii, and Their Effect on NO Release in Macrophages. Chem. Biodivers. 2008, 5, 758-763. [CrossRef]

65. Shin, J.M.; Lee, K.M.; Lee, H.J.; Yun, J.H.; Nho, C.W. Physalin A regulates the Nrf2 pathway through ERK and p38 for induction of detoxifying enzymes. BMC Complement. Altern. Med. 2019, 19, 101. [CrossRef] [PubMed]

66. Zhu, F.; Dai, C.; Fu, Y.; Loo, J.F.; Xia, D.; Gao, S.P.; Ma, Z.; Chen, Z. Physalin A exerts anti-tumor activity in non-small cell lung cancer cell lines by suppressing JAK/STAT3 signaling. Oncotarget 2016, 7, 9462-9476. [CrossRef] [PubMed]

67. He, H.; Zang, L.H.; Feng, Y.S.; Chen, L.X.; Kang, N.; Tashiro, S.; Onodera, S.; Qiu, F.; Ikejima, T. Physalin A induces apoptosis via p53-Noxa-mediated ROS generation, and autophagy plays a protective role against apoptosis through p38-NF-kappaB survival pathway in A375-S2 cells. J. Ethnopharmacol. 2013, 148, 544-555. [CrossRef]

68. He, H.; Zang, L.H.; Feng, Y.S.; Wang, J.; Liu, W.W.; Chen, L.X.; Kang, N.; Tashiro, S.; Onodera, S.; Qiu, F.; et al. Physalin A induces apoptotic cell death and protective autophagy in HT1080 human fibrosarcoma cells. J. Nat. Prod. 2013, 76, 880-888. [CrossRef]

69. He, H.; Feng, Y.S.; Zang, L.H.; Liu, W.W.; Ding, L.Q.; Chen, L.X.; Kang, N.; Hayashi, T.; Tashiro, S.; Onodera, S.; et al. Nitric oxide induces apoptosis and autophagy; autophagy down-regulates NO synthesis in physalin A-treated A375-S2 human melanoma cells. Food Chem. Toxicol. 2014, 71, 128-135. [CrossRef]

70. Kang, N.; Jian, J.F.; Cao, S.J.; Zhang, Q.; Mao, Y.W.; Huang, Y.Y.; Peng, Y.F.; Qiu, F.; Gao, X.M. Physalin A induces G2/M phase cell cycle arrest in human non-small cell lung cancer cells: Involvement of the p38 MAPK/ROS pathway. Mol. Cell. Biochem. 2016, 415, 145-155. [CrossRef] [PubMed]

71. Han, H.; Qiu, L.; Wang, X.; Qiu, F.; Wong, Y.; Yao, X. Physalins A and B inhibit androgen-independent prostate cancer cell growth through activation of cell apoptosis and downregulation of androgen receptor expression. Biol. Pharm. Bull. 2011, 34, 1584-1588. [CrossRef]

72. Cao, C.; Zhu, L.; Chen, Y.; Wang, C.H.; ShenTu, J.Z.; Zheng, Y.L. Physalin B induces G2/M cell cycle arrest and apoptosis in A549 human non-small-cell lung cancer cells by altering mitochondrial function. Anti-Cancer Drugs 2019, 30, 128-137. [CrossRef]

73. Hsu, C.C.; Wu, Y.C.; Farh, L.; Du, Y.C.; Tseng, W.K.; Wu, C.C.; Chang, F.R. Physalin B from Physalis angulata triggers the NOXA-related apoptosis pathway of human melanoma A375 cells. Food Chem. Toxicol. 2012, 50, 619-624. [CrossRef]

74. Ma, Y.M.; Han, W.; Li, J.; Hu, L.H.; Zhou, Y.B. Physalin B not only inhibits the ubiquitin-proteasome pathway but also induces incomplete autophagic response in human colon cancer cells in vitro. Acta Pharmacol. Sin. 2015, 36, 517-527. [CrossRef]

75. Vandenberghe, I.; Créancier, L.; Vispé, S.; Annereau, J.P.; Barret, J.M.; Pouny, I.; Samson, A.; Aussagues, Y.; Massiot, G.; Ausseil, F.; et al. Physalin B, a novel inhibitor of the ubiquitin-proteasome pathway, triggers NOXA-associated apoptosis. Biochem. Pharmacol. 2008, 76, 453-462. [CrossRef] 
76. Wang, A.; Wang, S.; Zhou, F.; Li, P.; Wang, Y.; Gan, L.; Lin, L. Physalin B induces cell cycle arrest and triggers apoptosis in breast cancer cells through modulating p53-dependent apoptotic pathway. Biomed. Pharmacother. 2018, 101, 334-341. [CrossRef]

77. Sun, Y.; Guo, T.; Zhang, F.B.; Wang, Y.N.; Liu, Z.; Guo, S.; Li, L. Isolation and characterization of cytotoxic withanolides from the calyx of Physalis alkekengi L. var franchetii. Bioorg. Chem. 2020, 96, 103614. [CrossRef] [PubMed]

78. Magalhães, H.I.; Veras, M.L.; Torres, M.R.; Alves, A.P.; Pessoa, O.D.; Silveira, E.R.; Costa-Lotufo LV de Moraes, M.O.; Pessoa, C. In-vitro and in-vivo antitumour activity of physalins B and D from Physalis angulata. J. Pharm. Pharmacol. 2006, 58, 235-241. [CrossRef] [PubMed]

79. Jacobo-Herrera, N.J.; Bremner, P.; Marquez, N.; Gupta, M.P.; Gibbons, S.; Muñoz, E.; Heinrich, M. Physalins from Witheringia solanacea as modulators of the NF-kappaB cascade. J. Nat. Prod. 2006, 69, 328-331. [CrossRef] [PubMed]

80. Chen, C.; Zhu, D.; Zhang, H.; Han, C.; Xue, G.; Zhu, T.; Luo, J.; Kong, L. YAP-dependent ubiquitination and degradation of beta-catenin mediates inhibition of Wnt signalling induced by physalin F in colorectal cancer. Cell Death Dis. 2018, 9, 591. [CrossRef] [PubMed]

81. Ooi, K.L.; Muhammad, T.S.; Sulaiman, S.F. Physalin F from Physalis minima L. triggers apoptosis-based cytotoxic mechanism in T-47D cells through the activation caspase-3- and c-myc-dependent pathways. J. Ethnopharmacol. 2013, 150, 382-388. [CrossRef]

82. Wu, S.Y.; Leu, Y.L.; Chang, Y.L.; Wu, T.S.; Kuo, P.C.; Liao, Y.R.; Teng, C.M.; Pan, S.L. Physalin F induces cell apoptosis in human renal carcinoma cells by targeting NF-kappaB and generating reactive oxygen species. PLoS ONE 2012, 7, e40727. [CrossRef]

83. Sun, J.L.; Jiang, Y.J.; Cheng, L. Two new physalin derivatives from Physalis alkekengi L. var. franchetii (Mast.) Makino. Nat. Prod. Res. 2021, 35, 203-206. [CrossRef] [PubMed]

84. Lin, H.; Zhang, C.; Zhang, H.; Xia, Y.Z.; Zhang, C.Y.; Luo, J.; Yang, L.; Kong, L.Y. Physakengose G induces apoptosis via EGFR/mTOR signaling and inhibits autophagic flux in human osteosarcoma cells. Phytomedicine 2018, 42, 190-198. [CrossRef] [PubMed]

85. Castro, D.P.; Moraes, C.S.; Gonzalez, M.S.; Ribeiro, I.M.; Tomassini, T.C.; Azambuja, P.; Garcia, E.S. Physalin B inhibits Trypanosoma cruzi infection in the gut of Rhodnius prolixus by affecting the immune system and microbiota. J. Insect Physiol. 2012, 58, 1620-1625. [CrossRef]

86. Garcia, E.S.; Castro, D.P.; Ribeiro, I.M.; Tomassini, T.C.; Azambuja, P. Trypanosoma rangeli: Effects of physalin B on the immune reactions of the infected larvae of Rhodnius prolixus. Exp. Parasitol. 2006, 112, 37-43. [CrossRef] [PubMed]

87. Meira, C.S.; Guimarães, E.T.; Bastos, T.M.; Moreira, D.R.; Tomassini, T.C.; Ribeiro, I.M.; Dos Santos, R.R.; Soares, M.B. Physalins B and F, seco-steroids isolated from Physalis angulata L., strongly inhibit proliferation, ultrastructure and infectivity of Trypanosoma cruzi. Parasitology 2013, 140, 1811-1821. [CrossRef]

88. Soares, M.B.; Brustolim, D.; Santos, L.A.; Bellintani, M.C.; Paiva, F.P.; Ribeiro, Y.M.; Tomassini, T.C.; Dos Santos, R.R. Physalins B, F and G, seco-steroids purified from Physalis angulata L., inhibit lymphocyte function and allogeneic transplant rejection. Int. Immunopharmacol. 2006, 6, 408-414. [CrossRef]

89. Pinto, L.A.; Meira, C.S.; Villarreal, C.F.; Vannier-Santos, M.A.; de Souza, C.V.; Ribeiro, I.M.; Tomassini, T.C.; Galvão-Castro, B.; Soares, M.B.; Grassi, M.F. Physalin F, a seco-steroid from Physalis angulata L., has immunosuppressive activity in peripheral blood mononuclear cells from patients with HTLV1-associated myelopathy. Biomed. Pharmacother. 2016, 79, 129-134. [CrossRef]

90. Yu, Y.; Sun, L.; Ma, L.; Li, J.; Hu, L.; Liu, J. Investigation of the immunosuppressive activity of physalin $\mathrm{H}$ on $\mathrm{T}$ lymphocytes. Int. Immunopharmacol. 2010, 10, 290-297. [CrossRef]

91. Yang, H.; Han, S.; Zhao, D.; Wang, G. Adjuvant effect of polysaccharide from fruits of Physalis alkekengi L. in DNA vaccine against systemic candidiasis. Carbohydr. Polym. 2014, 109, 77-84. [CrossRef]

92. Helvac1, S.; Kökdil, G.; Kawai, M.; Duran, N.; Duran, G.; Güvenç, A. Antimicrobial activity of the extracts and physalin D from Physalis alkekengi and evaluation of antioxidant potential of physalin D. Pharm. Biol. 2010, 48, 142-150. [CrossRef]

93. Yang, Y.K.; Xie, S.D.; Xu, W.X.; Nian, Y.; Liu, X.L.; Peng, X.R.; Ding, Z.T.; Qiu, M.H. Six new physalins from Physalis alkekengi var. franchetii and their cytotoxicity and antibacterial activity. Fitoterapia 2016, 112, 144-152. [CrossRef]

94. Januário, A.H.; Filho, E.R.; Pietro, R.C.; Kashima, S.; Sato, D.N.; França, S.C. Antimycobacterial physalins from Physalis angulata L. (Solanaceae). Phytother. Res. 2002, 16, 445-448. [CrossRef]

95. Li, X.; Zhang, C.; Wu, D.; Tang, L.; Cao, X.; Xin, Y. In vitro effects on intestinal bacterium of physalins from Physalis alkekengi var. Francheti. Fitoterapia 2012, 83, 1460-1465. [CrossRef]

96. Zhang, C.Y.; Luo, J.G.; Liu, R.H.; Lin, R.; Yang, M.H.; Kong, L.Y. 1H NMR spectroscopy-guided isolation of new sucrose esters from Physalis alkekengi var. franchetii and their antibacterial activity. Fitoterapia 2016, 114, 138-143. [CrossRef] [PubMed]

97. Zhang, C.Y.; Luo, J.G.; Liu, R.H.; Lin, R.; Yang, M.H.; Kong, L.Y. Physakengoses K-Q, seven new sucrose esters from Physalis alkekengi var. franchetii. Carbohydr. Res. 2017, 449, 120-124. [CrossRef] [PubMed]

98. Guimarães, E.T.; Lima, M.S.; Santos, L.A.; Ribeiro, I.M.; Tomassini, T.B.; dos Santos, R.R.; dos Santos, W.L.; Soares, M.B. Activity of physalins purified from Physalis angulata in in vitro and in vivo models of cutaneous leishmaniasis. J. Antimicrob. Chemother. 2009, 64, 84-87. [CrossRef]

99. Bao, C.L. Curative Effect of Chinese Physalis Alkekeng on Mice Allergic Asthuma; Yanbian University: Yanji, China, 2008.

100. Hu, X.F.; Zhang, Q.; Zhang, P.P.; Sun, L.J.; Liang, J.C.; Morris-Natschke, S.L.; Chen, Y.; Lee, K.H. Evaluation of in vitro/in vivo anti-diabetic effects and identification of compounds from Physalis alkekengi. Fitoterapia 2018, 127, 129-137. [CrossRef]

101. Guo, Y.; Li, S.; Li, J.; Ren, Z.; Chen, F.; Wang, X. Anti-hyperglycemic activity of polysaccharides from calyx of Physalis alkekengi var. franchetii Makino on alloxan-induced mice. Int. J. Biol. Macromol. 2017, 99, 249-257. [CrossRef] 
102. Zhang, Q.; Hu, X.F.; Xin, M.M.; Liu, H.B.; Sun, L.J.; Morris-Natschke, S.L.; Chen, Y.; Lee, K.H. Antidiabetic potential of the ethyl acetate extract of Physalis alkekengi and chemical constituents identified by HPLC-ESI-QTOF-MS. J. Ethnopharmacol. 2018, 225, 202-210. [CrossRef] [PubMed]

103. Yang, Y.; Ding, Z.; Wang, Y.; Zhong, R.; Feng, Y.; Xia, T.; Xie, Y.; Yang, B.; Sun, X.; Shu, Z. Systems pharmacology reveals the mechanism of activity of Physalis alkekengi L. var. franchetii against lipopolysaccharide-induced acute lung injury. J. Cell. Mol. Med. 2020, 24, 5039-5056. [CrossRef]

104. Zhang, W.; Bai, S.S.; Zhang, Q.; Shi, R.L.; Wang, H.C.; Liu, Y.C.; Ni, T.J.; Wu, Y.; Yao, Z.Y.; Sun, Y.; et al. Physalin B reduces A $\beta$ secretion through down-regulation of BACE1 expression by activating FoxO1 and inhibiting STAT3 phosphorylation. Chin. J. Nat. Med. 2021, 19, 732-740. [CrossRef]

105. Sá, M.S.; de Menezes, M.N.; Krettli, A.U.; Ribeiro, I.M.; Tomassini, T.C.; dos Santos, R.R.; de Azevedo, W.F.J.; Soares, M.B. Antimalarial activity of physalins B, D, F, and G. J. Nat. Prod. 2011, 74, 2269-2272. [CrossRef] [PubMed]

106. Yang, Y.; Piao, X.; Zhang, M.; Wang, X.; Xu, B.; Zhu, J.; Fang, Z.; Hou, Y.; Lu, Y.; Yang, B. Bioactivity-guided fractionation of the triglyceride-lowering component and in vivo and in vitro evaluation of hypolipidemic effects of Calyx seu Fructus Physalis. Lipids Health Dis. 2012, 11, 38. [CrossRef]

107. Yang, Y.; Chen, B.; Liang, K.L.; Su, J.; Chen, S.H.; Lv, G.Y. Relaxation effect of buddleoside combined with luteolin on isolated vessels in vivo and its mechanism. China J. Chin. Mater. Med. 2017, 42, 1370-1375.

108. Yan, Q.; Li, Y.; Yan, J.; Zhao, Y.; Liu, Y.; Liu, S. Effects of luteolin on regulatory proteins and enzymes for myocyte calcium circulation in hypothermic preserved rat heart. Exp. Ther. Med. 2018, 15, 1433-1441. [CrossRef]

109. Tariq, A.; Adnan, M.; Amber, R.; Pan, K.; Mussarat, S.; Shinwari, Z.K. Ethnomedicines and anti-parasitic activities of Pakistani medicinal plants against Plasmodia and Leishmania parasites. Ann. Clin. Microbiol. Antimicrob. 2016, 15, 52. [CrossRef]

110. Guimarães, E.T.; Lima, M.S.; Santos, L.A.; Ribeiro, I.M.; Tomassini, B.C.; Santos, R.R.; Santos, L.C.; Soares, B.P. Effects of secosteroids purified from Physalis angulata L., Solanaceae, on the viability of Leishmania sp.. Rev. Bras. Farmacogn. 2010, 20, 945-949. [CrossRef]

111. Tan, X.; Jin, P.; Feng, L.; Song, J.; Sun, E.; Liu, W.; Shu, L.; Jia, X. Protective effect of luteolin on cigarette smoke extract-induced cellular toxicity and apoptosis in normal human bronchial epithelial cells via the Nrf2 pathway. Oncol. Rep. 2014, 31, 1855-1862. [CrossRef]

112. Guo, Y.; Liu, H.; Ding, L.; Oppong, M.; Pan, G.; Qiu, F. LC-MS/MS method for simultaneous determination of flavonoids and physalins in rat plasma: Application to pharmacokinetic study after oral administration of Physalis alkekengi var. franchetii (Chinese lantern) extract. Biomed. Chromatogr. 2017, 31, e3970. [CrossRef]

113. Zheng, Y.; Chen, Y.; Ren, Y.; Luan, L.; Wu, Y. An ultra-pressure liquid chromatography-Tandem mass spectrometry method for the simultaneous determination of three physalins in rat plasma and its application to pharmacokinetic study of Physalis alkekengi var. franchetii (Chinese lantern) in rats. J. Pharm. Biomed. Anal. 2012, 58, 94-101. [CrossRef]

114. Shen, X.; Zheng, Y.L.; ShenTu, J.Z. Pharmacokinetic of physalin L in rat plasma. Chin. J. Mod. Appl. Pharm. 2017, 34, 1663-1667.

115. Zheng, Y.; Lin, M.; Hu, X.; Zhai, Y.; Zhang, Q.; Lou, Y.; ShenTu, J.; Wu, L. Simultaneous pharmacokinetics and stability studies of physalins in rat plasma and intestinal bacteria culture media using liquid chromatography with mass spectrometry. J. Sep. Sci. 2018, 41, 1781-1790. [CrossRef]

116. Zheng, Y.; Chen, J.; Liu, L.; Liang, X.; Hong, D. In vivo pharmacokinetics of and tissue distribution study of physalin B after intravenous administration in rats by liquid chromatography with tandem mass spectrometry. Biomed. Chromatogr. 2016, 30, 1278-1284. [CrossRef]

117. Fang, L.; Chai, H.B.; Castillo, J.J.; Soejarto, D.D.; Farnsworth, N.R.; Cordell, G.A.; Pezzuto, J.M.; Kinghorn, A.D. Cytotoxic constituents of Brachistus stramoniifolius. Phytother. Res. 2003, 17, 520-523. [CrossRef]

118. Wu, Y.; Zheng, Y.; Chen, N.; Luan, L.; Liu, X. Plasma pharmacokinetics and tissue distribution study of physalin D in rats by ultra-pressure liquid chromatography with tandem mass spectrometry. J. Chromatogr. B 2011, 879, 443-448. [CrossRef]

119. Feng, X.; Huo, X.; Liu, H.; Chai, L.; Ding, L.; Qiu, F. Identification of absorbed constituents and in vivo metabolites in rats after oral administration of Physalis alkekengi var. franchetii by ultra-high-pressure liquid chromatography quadrupole time-of-flight mass spectrometry. Biomed. Chromatogr. 2018, 32, e4121. [CrossRef]

120. Feng, X.; Liu, H.; Chai, L.; Ding, L.; Pan, G.; Qiu, F. Metabolic profiles of physalin A in rats using ultra-high performance liquid chromatography coupled with quadrupole time-of-flight tandem mass spectrometry. J. Chromatogr. B 2017, 1046, 102-109. [CrossRef]

121. Zheng, Y.; Cao, C.; Lin, M.; Zhai, Y.; Ge, Z.; ShenTu, J.; Wu, L.; Hu, X. Identification and quantitative analysis of physalin D and its metabolites in rat urine and feces by liquid chromatography with triple quadrupole time-of-flight mass spectrometry. J. Sep. Sci. 2017, 40, 2355-2365. [CrossRef] [PubMed]

122. Castro, D.P.; Figueiredo, M.B.; Ribeiro, I.M.; Tomassini, T.C.; Azambuja, P.; Garcia, E.S. Immune depression in Rhodnius prolixus by seco-steroids, physalins. J. Insect Physiol. 2008, 54, 555-562. [CrossRef] [PubMed]

123. Otake, Y.; Hsieh, F.; Walle, T. Glucuronidation versus oxidation of the flavonoid galangin by human liver microsomes and hepatocytes. Drug Metab. Dispos. 2002, 30, 576-581. [CrossRef] [PubMed]

124. Chen, Z.; Zheng, S.; Li, L.; Jiang, H. Metabolism of flavonoids in human: A comprehensive review. Curr. Drug Metab. 2014, 15, 48-61. [CrossRef] [PubMed] 
125. Day, A.J.; DuPont, M.S.; Ridley, S.; Rhodes, M.; Rhodes, M.J.; Morgan, M.R.; Williamson, G. Deglycosylation of flavonoid and isoflavonoid glycosides by human small intestine and liver beta-glucosidase activity. FEBS Lett. 1998, 436, 71-75. [CrossRef]

126. Shu, Z.; Tang, Y.; Yang, Y.; Ding, Z.; Zhong, R.; Xia, T.; Li, X.; Zheng, C.; Wen, Z.; Li, W.; et al. Two new 3-hexenol glycosides from the calyces of Physalis alkekengi var. franchetii. Nat. Prod. Res. 2021, 35, 1274-1280. [CrossRef] [PubMed]

127. Sun, J.M.; He, J.X.; Huang, M.; Hu, H.X.; Xu, L.T.; Fang, K.L.; Wang, X.N.; Shen, T. Two new physalins from Physalis alkekengi L. var. franchetii (Mast.) Makino. Nat. Prod. Res. 2021, 1-7. [CrossRef] [PubMed]

128. Yoritate, M.; Morita, Y.; Gemander, M.; Morita, M.; Yamashita, T.; Sodeoka, M.; Hirai, G. Synthesis of DFGH-Ring Derivatives of Physalins via One-Pot Construction of GH-Ring and Evaluation of Their NF-kB-Inhibitory Activity. Org. Lett. 2020, 22, 8877-8881. [CrossRef] 